\title{
EXISTENCE AND REGULARITY RESULTS FOR SOME PARABOLIC EQUATIONS WITH DEGENERATE COERCIVITY
}

\author{
Fengquan Li \\ Dalian University of Technology, School of Mathematical Sciences \\ Dalian, 116024, P. R. China; fqli@dlut.edu.cn
}

\begin{abstract}
In this paper, we study the existence and regularity results for some parabolic equations with degenerate coercivity.
\end{abstract}

\section{Introduction and statement of the main results}

This paper will deal with the following problem

$$
\begin{cases}\frac{\partial u}{\partial t}+A u=f & \text { in } Q \\ u=0 & \text { on } \partial \Omega \times(0, T), \\ u(x, 0)=0 & \text { in } \Omega\end{cases}
$$

where

$$
A u=-\operatorname{div}(a(x, t, u) D u),
$$

$f \in L^{m}(Q), m \geq 1, \Omega$ is an open bounded subset of $\mathbf{R}^{N}(N \geq 2), T$ is a positive constant, $Q=\Omega \times(0, T)$ with the lateral boundary $\partial \Omega \times(0, T)$.

Let $a: Q \times \mathbf{R} \rightarrow \mathbf{R}$ be a Carathéodory function satisfying for almost every $(x, t) \in Q$ and every $s \in R$,

$$
\frac{\alpha}{(1+|s|)^{\theta}} \leq a(x, t, s) \leq \beta
$$

and

$$
0 \leq \theta<1+\frac{2}{N}
$$

where $\alpha, \beta$ are two positive constants.

If (1.1) holds true, the differential operator $A(u)$ is not coercive as $u$ becomes large. This shows that the classical methods (see [22]) can't be applied to prove the existence of solutions to problem $(P)$ even if the data $f$ is sufficiently regular. The goal in this paper is to study the problem $(P)$ under the assumptions of (1.1)-(1.2). The proof is essentially based on the approximate problems $\left(P_{n}\right)$ with some nondegenerate coercivity and a priori estimates on the weak solutions of these problems. Similar problem to elliptic equations has already been studied in [13] (see also [1, 2, 9, 10, 18, 19]). Recently, Porzio and Pozio in [24] have discussed the case of $f \equiv 0, u(x, 0)=u_{0} \neq 0$.

Now we state the main results of this paper.

doi:10.5186/aasfm.2012.3738

2010 Mathematics Subject Classification: Primary 35K65, 35K55.

Key words: Existence and regularity, parabolic equations, degenerate coercivity. 
Theorem 1.1. Under the hypotheses (1.1)-(1.2), if $f \in L^{m}(Q)$ with $m>\frac{N}{2}+1$, then there exists a bounded weak solution $u \in L^{2}\left(0, T ; H_{0}^{1}(\Omega)\right) \cap L^{\infty}(Q)$ to problem $(P)$.

Remark 1.1. This result doesn't depend on $\theta$ and is similar to the one obtained in the coercive case. This seems to be natural, since if one looks for bounded solutions, the lack of coercivity of the differential operator $A$ (which is caused by unbounded functions) "disappears". Moreover, if $\theta=0$, this result has been proved by Aronson and Serrin (see [4]).

Theorem 1.2. Under the hypotheses (1.1)-(1.2), if $f \in L^{m}(Q)$ with $m=\frac{N}{2}+1$, then there exists a weak solution $u \in L^{2}\left(0, T ; H_{0}^{1}(\Omega)\right) \cap L^{r}(Q)$ to problem $(P)$ with $2 \leq r<+\infty$.

Remark 1.2. Theorem 1.2 gives the result in the limit case $m=\frac{N}{2}+1$ for parabolic equations. As far as I know, I haven't found other works dealing with the limit case for parabolic equations even if $\theta=0$.

Theorem 1.3. Under the hypotheses (1.1)-(1.2), if $f \in L^{m}(Q)$ with $m$ such that

$$
\frac{2(N+2+\theta)}{N+4-(N-2) \theta} \leq m<\frac{N}{2}+1
$$

then there exists a weak solution $u \in L^{2}\left(0, T ; H_{0}^{1}(\Omega)\right) \cap L^{r}(Q)$ of problem $(P)$ with

$$
r=\frac{m[N(1-\theta)+2]}{N+2-2 m} \text {. }
$$

Remark 1.3. If $0 \leq \theta<\frac{2}{N-1}$, then $\frac{2(N+2+\theta)}{N+4-(N-2) \theta}<2$, and in this case the function $f$ is not in general in $L^{2}\left(0, T ; H^{-1}(\Omega)\right)$. If $\frac{2}{N-1} \leq \theta<1+\frac{2}{N}$, then $\frac{2(N+2+\theta)}{N+4-(N-2) \theta} \geq 2$, and in this case the function $f$ belongs to the space $L^{2}\left(0, T ; H^{-1}(\Omega)\right)$. In any case, we always hope to find a weak solution $u \in L^{2}\left(0, T ; H_{0}^{1}(\Omega)\right)$ of problem $(P)$, but the weak solution $u$ can not be directly obtained from the equation because $u$ may be unbounded. Here we find the weak solution by means of a priori estimates in $L^{r}(Q)$ which is then used to prove that $|D u|$ belongs to $L^{2}(Q)$.

Remark 1.4. If $a$ is independent of $s$ or $\theta=0$ in (1.1), the previous theorem has been proved in [20] and [8], respectively (see [12]).

Theorem 1.4. Under the hypotheses (1.1)-(1.2), if $f \in L^{m}(Q)$ with $m>1$ such that

$$
\frac{N+2+\theta}{N+3-\theta(N-1)}<m<\frac{2(N+2+\theta)}{N+4-(N-2) \theta}
$$

then there exists a function $u \in L^{q}\left(0, T ; W_{0}^{1, q}(\Omega)\right) \cap L^{r}(Q)$ with

$$
q=\frac{m[N(1-\theta)+2]}{N+1-(1+\theta)(m-1)}, \quad r=\frac{m[N(1-\theta)+2]}{N+2-2 m}
$$

and satisfies problem $(P)$ in the sense of distributions, that is, for any $\psi$ in $C^{\infty}(\bar{Q})$ which is zero in a neighborhood of $\partial \Omega \times(0, T)$ and $\Omega \times\{T\}$ such that

$$
-\int_{Q} u \psi^{\prime} d x d t+\int_{Q} a(x, t, u) D u D \psi d x d t=\int_{Q} f \psi d x d t .
$$


Remark 1.5. The lower bound for $m$ in (1.5) is due to the fact that $q$ must not be smaller than 1. The upper bound for $m$ in (1.5) implies $q<2$. In the above theorem, we also suppose $m>1$ because if $0 \leq \theta<\frac{1}{N}$, then $\frac{N+2+\theta}{N+3-\theta(N-1)}<1$. If $\theta=0$, the result of Theorem 1.4 coincides with the classical regularity result for parabolic equations with coercivity (see [11]).

In the above theorems, the solutions belong to some Sobolev space. If the summability conditions on $f$ will be weaken, the gradient of $u$ may no longer be in $L^{1}(\Omega)$. To overcome this difficulty, we may give the meaning to solutions of problem $(P)$ by using the concept of entropy solutions (for elliptic equations, see [6], for parabolic equations, see $[3,17,21,23,25,26])$.

For $k>0$, let

$$
T_{k}(s)=\min \{k, \max \{-k, s\}\}, \quad S_{k}(s)=\int_{0}^{s} T_{k}(\tau) d \tau, \forall s \in R .
$$

Theorem 1.5. Under the hypotheses (1.1)-(1.2), if $f \in L^{m}(Q)$ with

$$
1 \leq m \leq \max \left\{\frac{N+2+\theta}{N+3-\theta(N-1)}, 1\right\}
$$

then there exists an entropy solution $u$ to problem $(P)$ in the sense of Definition 2.1 with

$$
u \in \mathcal{M}^{r}(Q), \quad r=\frac{m[N(1-\theta)+2]}{N+2-2 m}
$$

and

$$
|D u| \in \mathcal{M}^{q}(Q), \quad q=\frac{m[N(1-\theta)+2]}{N+1-(1+\theta)(m-1)},
$$

where $\mathcal{M}^{r}(Q)$ and $\mathcal{M}^{q}(Q)$ are Marcinkiewicz spaces defined in Definition 2.2.

Remark 1.6. If $0 \leq \theta<\frac{1}{N}$, then (1.9) becomes $m=1$, thus $r=\frac{N(1-\theta)+2}{N}>$ $1, q=\frac{N(1-\theta)+2}{N+1}>1$. By the embedding theorems between Marcinkiewicz and Lebesgue spaces, we can deduce that $u$ belongs to $L^{p}\left(0, T ; W_{0}^{1, p}(\Omega)\right)$ for every $1 \leq$ $p<q=\frac{N(1-\theta)+2}{N+1}$. If in particular $\theta=0$, this is the same result obtained in [11] and [14] for parabolic equations with measure data (see also [3, 5, 15, 26]).

Remark 1.7. If $\frac{1}{N} \leq \theta<1+\frac{2}{N}$, then (1.9) becomes $1 \leq m \leq \frac{N+2+\theta}{N+3-\theta(N-1)}$ and $q$ must be smaller than 1 . It is not possible to deduce that $|D u|$ belongs to some Sobolev space even if $1<m \leq \frac{N+2+\theta}{N+3-\theta(N-1)}$. Thus Theorem 1.5 shows that the regularity of solutions to parabolic equations with degenerate coercivity is essentially different from that of parabolic equations with coercivity, since the solutions belong to some Sobolev space for the latter so long as $m>1$ (see $[11,15]$ ).

Remark 1.8. Here the upper bound on $\theta$ in (1.2) is $1+\frac{2}{N}$, but 1 for elliptic equations (see $[1,2,9,10,13,18,19])$. This is due to two different types of partial differential equations. The condition $\theta<1+\frac{2}{N}$ implies that the assumpations (1.3) and (1.5) hold, otherwise (1.3) and (1.5) become empty.

Theorem 1.6. Under the hypothesis (1.1), let $f=0, u_{0} \in L^{d}(\Omega), d_{0} \equiv$ $\min \left\{d_{1}, d_{2}\right\}, d_{1} \equiv \frac{\theta+2}{2}, d_{2} \equiv \frac{N(\theta+1)}{N+1}$ where $\theta$ is a nonnegative constant. 
(i) Suppose that $0 \leq \theta \leq \frac{2}{N-1}$. If $1 \leq d \leq \frac{N \theta}{2}$, then there exists an entropy solution $u$ to problem $(P)$ in the sense of Definition 2.1 with

$$
u \in L^{\infty}\left(0, T ; L^{d}(\Omega)\right)
$$

and

$$
|D u| \in \mathcal{M}^{q}(Q), \quad q=\frac{2 d}{\theta+2},
$$

where $\mathcal{M}^{q}(Q)$ is Marcinkiewicz spaces defined in Definition 2.2. If $\max \left\{1, \frac{N \theta}{2}\right\} \leq$ $d \leq d_{0}$, then (1.12) still holds and

$$
|D u| \in \mathcal{M}^{q}(Q), \quad q=\frac{(N+2) d-N \theta}{N+d} .
$$

(ii) Suppose that $\frac{2}{N-1} \leq \theta$. If $1 \leq d \leq d_{0}$, then there exists an entropy solution $u$ to problem $(P)$ in the sense of Definition 2.1 with

$$
u \in L^{\infty}\left(0, T ; L^{d}(\Omega)\right)
$$

and

$$
|D u| \in \mathcal{M}^{q}(Q), \quad q=\frac{2 d}{\theta+2} .
$$

Remark 1.9. Porzio and Pozio in Theorem 2.9 of [24] have discussed the case of $f=0, u_{0} \in L^{d}(\Omega), d>\max \left\{1, d_{0}\right\}$, and in Theorem 2.10 of [24], they only consider the case of $d>\frac{N \theta}{2}$. Here Theorem 1.6 discussed all cases of $1 \leq d \leq d_{0}$. Therefore, Theorem 1.6 is a complement of [24]. Furthermore, as $d=1$, Theorem 1.6 shows more regular solution than Theorem 2.13 in [24] and don't need the smaller conditions of $\theta$.

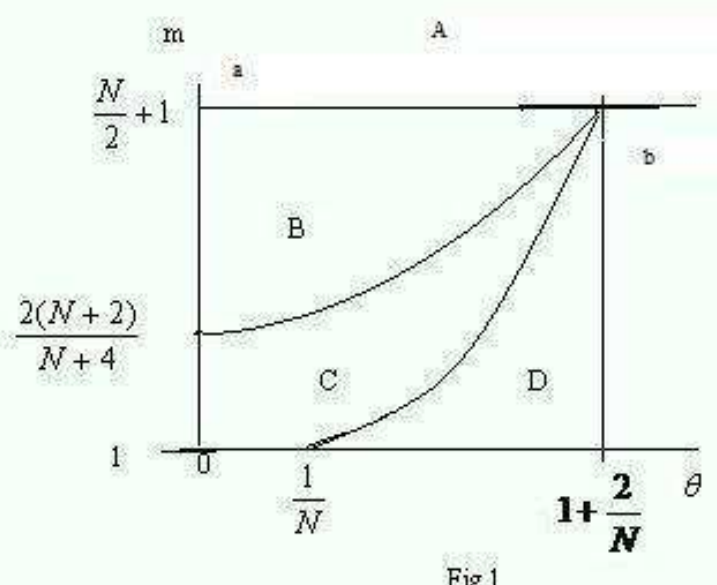

Fig.1

Figure 1. This figure gives different results obtained in this paper dependent of $m$ and $\theta$. If $(m, \theta)$ lies in the different regions $A$, line segment $a b, B, C, D$, these results are obtained in Theorems 1.1-1.5, respectively. 
This paper is organized as follows. In Section 2, some preliminary results and a priori estimates will be given. In Section 3, we will finish the proofs of Theorems 1.11.6 .

\section{Some preliminary results and a priori estimates}

Before we prove Theorem 1.1-1.6, we need some preliminary results.

Definition 2.1. A measurable function $u \in L^{\infty}\left(0, T ; L^{1}(\Omega)\right)$ will be called an entropy solution to problem $(P)$ if $T_{k}(u) \in L^{2}\left(0, T ; H_{0}^{1}(\Omega)\right)$ for every $k>0$, and if

$$
\begin{gathered}
\int_{\Omega} S_{k}(u(t)-\phi(t)) d x \in C[0, T] \\
\int_{\Omega} S_{k}(u(T)-\phi(T)) d x-\int_{\Omega} S_{k}(-\phi(0)) d x+\int_{0}^{T}\left\langle\phi_{t}, T_{k}(u-\phi)\right\rangle d t \\
+\int_{Q} a(x, t, u) D u D T_{k}(u-\phi) d x d t \leq \int_{Q} f T_{k}(u-\phi) d x d \tau,
\end{gathered}
$$

for every $k>0$ and $\phi \in L^{2}\left(0, T ; H_{0}^{1}(\Omega)\right) \cap L^{\infty}(Q)$ such that $\phi_{t} \in L^{2}\left(0, T ; H^{-1}(\Omega)\right)+$ $L^{1}(Q)$.

Similarly to Lemma 2.1 in [6], we also have

Lemma 2.1. For every $k>0$, if $T_{k}(u) \in L^{2}\left(0, T ; H_{0}^{1}(\Omega)\right)$, then there exists a unique measurable function $v: Q \longmapsto \mathbf{R}^{N}$ such that

$$
D T_{k}(u)=v \chi_{\{|u|<k\}} \text { a.e. in } Q,
$$

where $\chi_{\{|u|<k\}}$ denotes the characteristic function over the set $\{|u|<k\}$. Defining the derivative $D u$ of $u$ as the unique function $v$ which satisfies the above equality. Furthermore, $u \in L^{2}\left(0, T ; H_{0}^{1}(\Omega)\right)$ if and only if $v \in L^{2}(Q)$, and then $v \equiv D u$ in the usual weak sense.

Proof. The proof of Lemma 2.1 is the same as that of Lemma 2.1 in [6], we omit the details.

Definition 2.2. $[7,26]$ For $0<q<+\infty$, the set of all measurable functions $u: Q \rightarrow R$ such that the functional $[u]_{q}=\sup _{k>0} k$ meas $\{(x, t) \in Q:|u(x, t)|>k\}^{\frac{1}{q}}$ is finite is called a Marcinkiewicz space and is denoted by $\mathcal{M}^{q}(Q)$.

One can deduce that $\mathcal{M}^{q}(Q) \subset \mathcal{M}^{r}(Q)$ for $r<q$, and $L^{q}(Q) \subset \mathcal{M}^{q}(Q) \subset L^{r}(Q)$ for $r<q($ see $[13,15])$.

We also recall a consequence of the Gagliardo-Nirenberg embedding theorem.

Lemma 2.2. [16, Proposition 3.1] Let $v \in L^{h}\left(0, T ; W_{0}^{1, h}(\Omega)\right) \cap L^{\infty}\left(0, T ; L^{\varrho}(\Omega)\right)$, $\varrho, h \geq 1$. Then $v$ belongs to $L^{q_{0}}(Q)$, where $q_{0}=h \frac{(N+\varrho)}{N}$, and there exists a positive constant $C$ depending only on $N, h, \varrho$ such that

$$
\int_{Q}|v(x, t)|^{q_{0}} d x d t \leq C\left(\operatorname{ess} \sup _{0<t<T} \int_{\Omega}|v(x, t)|^{\varrho} d x\right)^{\frac{h}{N}} \int_{Q}|D v(x, t)|^{h} d x d t .
$$

The following lemma gives another version of Lemma 3.2 in [13]. 
Lemma 2.3. Let $v$ be a measurable function in $\mathcal{M}^{\mu}(Q)$ for $\mu>0$, and assume that there exist two nonegative constants $\nu>\gamma$ such that

$$
\int_{Q}\left|D T_{k}(v)\right|^{2} d x d t \leq M(1+k)^{\gamma} k^{\nu-\gamma}, \quad \forall k>0,
$$

where $M$ is a positive constant independent of $k$. Then $|D v| \in \mathcal{M}^{\delta}(Q)$, with $\delta=\frac{2 \mu}{\mu+\nu}$.

Proof. Let $l$ be a fixed positive number. We have for every $k>0$,

$$
\operatorname{meas}\{|D v|>l\} \leq \operatorname{meas}\{|v|>k\}+\operatorname{meas}\{|D v|>l,|v| \leq k\} .
$$

Moreover,

$$
\operatorname{meas}\{|D v|>l,|v| \leq k\} \leq \frac{1}{l^{2}} \int_{Q}\left|D T_{k}(v)\right|^{2} d x d t \leq M \frac{(1+k)^{\gamma} k^{\nu-\gamma}}{l^{2}} .
$$

If $k>1$, then the above inequality turns into

$$
\operatorname{meas}\{|D v|>l,|v| \leq k\} \leq 2^{\gamma} M \frac{k^{\nu}}{l^{2}} .
$$

By Definition 2.2 and $v \in \mathcal{M}^{\mu}(Q)$, then there exists a positive constant $M_{1}$ independent of $k$ such that

$$
\operatorname{meas}\{|v|>k\} \leq \frac{M_{1}}{k^{\mu}}
$$

Hence, we have

$$
\operatorname{meas}\{|D v|>l\} \leq M_{2}\left(\frac{k^{\nu}}{l^{2}}+\frac{1}{k^{\mu}}\right),
$$

where $M_{2}=\max \left\{2^{\gamma} M, M_{1}\right\}$. Minimizing with respect to $k$, we easily prove that as $k=\left(\frac{\mu}{\nu}\right)^{\frac{1}{\mu+\nu}} l^{\frac{2}{\mu+\nu}}$, the minimum value of the right side term in the above inequality is achieved, and we get

$$
\operatorname{meas}\{|D v|>l\} \leq \frac{M_{3}}{l^{\delta}},
$$

where $M_{3}$ is a positive constant independent of $l$. However, the above conclusion is obtained under the assumpation $k>1$, that is $l>\left(\frac{\nu}{\mu}\right)^{\frac{1}{2}}$. If $l \leq\left(\frac{\nu}{\mu}\right)^{\frac{1}{2}}$, since $Q$ is bounded, the above inequality obviously holds. This inequality and Definition 2.2 yield $|D v| \in \mathcal{M}^{\delta}(Q)$.

For convenience, we will denote the Lebesgue measure of any measurable set $E$ by $|E|$ in the follwing text.

In order to discuss problem $(P)$, we need consider the approximate problems.

$$
\begin{cases}\frac{\partial u_{n}}{\partial t}-\operatorname{div}\left(a\left(x, t, T_{n}\left(u_{n}\right)\right) D u_{n}\right)=f_{n} & \text { in } Q, \\ u_{n}=0 & \text { on } \partial \Omega \times(0, T), \\ u_{n}(x, 0)=0 & \text { in } \Omega,\end{cases}
$$

where $f_{n} \in \mathcal{D}(Q)$ and satisfy

$$
\begin{aligned}
& \left\|f_{n}\right\|_{L^{m}(Q)} \leq\|f\|_{L^{m}(Q)}, \quad \forall n, \\
& f_{n} \rightarrow f \text { strongly in } L^{m}(Q) .
\end{aligned}
$$


Then from the well-known result of [22], there exists at least a solution $u_{n} \in C([0, T]$; $\left.L^{2}(\Omega)\right) \cap L^{2}\left(0, T ; H_{0}^{1}(\Omega)\right)$ to problem $\left(P_{n}\right)$ such that $u_{n}^{\prime} \in L^{2}\left(0, T ; H^{-1}(\Omega)\right)$ and satisfies

$$
\int_{Q} u_{n}^{\prime} \psi d x d t+\int_{Q} a\left(x, t, T_{n}\left(u_{n}\right)\right) D u_{n} D \psi d x d t=\int_{Q} f_{n} \psi d x d t
$$

for any $\psi \in L^{2}\left(0, T ; H_{0}^{1}(\Omega)\right)$ and $u_{n}(x, 0)=0$.

We have a priori estimates on $u_{n}$ as follows.

Lemma 2.4. Assume that $m>\frac{N}{2}+1$ in (2.3)-(2.4) and (1.1)-(1.2) hold. Then for every solution $u_{n}$ of problem $\left(P_{n}\right)$, there exists a positive constant $C_{1}$ independent of $n$ such that

$$
\begin{aligned}
\left\|u_{n}\right\|_{L^{\infty}(Q)} & \leq C_{1}, \\
\left\|u_{n}\right\|_{L^{2}\left(0, T ; H_{0}^{1}(\Omega)\right)} & \leq C_{1}, \\
\left\|u_{n}^{\prime}\right\|_{L^{2}\left(0, T ; H^{-1}(\Omega)\right)+L^{m}(Q)} & \leq C_{1} .
\end{aligned}
$$

Proof. (i) Suppose that $\theta>0$. Let $G_{k}(s)=s-T_{k}(s), \forall s \in R, k>0$. For all $\tau \in(0, T]$, using $G_{k}\left(u_{n}(x, t)\right) \chi_{(0, \tau)}(t)$ as a test function for problem $\left(P_{n}\right)$, where $\chi_{(0, \tau)}$ denotes the characteristic function of $(0, \tau)$ in $(0, T]$, we get

$$
\begin{aligned}
& \int_{0}^{\tau} \int_{\Omega} u_{n}^{\prime} G_{k}\left(u_{n}\right) d x d t+\int_{0}^{\tau} \int_{\Omega} a\left(x, t, T_{n}\left(u_{n}\right)\right) D u_{n} D G_{k}\left(u_{n}\right) d x d t \\
& =\int_{0}^{\tau} \int_{\Omega} f_{n} G_{k}\left(u_{n}\right) d x d t .
\end{aligned}
$$

Let

$$
A_{k}(t)=\left\{x \in \Omega:\left|u_{n}(x, t)\right|>k\right\} .
$$

By (1.1) and Hölder's inequality, we obtain

$$
\begin{aligned}
& \frac{1}{2} \int_{A_{k}(\tau)}\left|G_{k}\left(u_{n}(\tau)\right)\right|^{2} d x+\alpha \int_{0}^{\tau} \int_{A_{k}(t)} \frac{\left|D u_{n}\right|^{2}}{\left(1+\left|u_{n}\right|\right)^{\theta}} d x d t \\
& \leq \frac{1}{2} \int_{A_{k}(\tau)}\left|G_{k}\left(u_{n}(\tau)\right)\right|^{2} d x+\alpha \int_{0}^{\tau} \int_{A_{k}(t)} \frac{\left|D u_{n}\right|^{2}}{\left(1+\left|T_{n}\left(u_{n}\right)\right|\right)^{\theta}} d x d t \\
& \leq\left\|f_{n}\right\|_{L^{m}(Q)}\left(\int_{0}^{\tau} \int_{A_{k}(t)}\left|G_{k}\left(u_{n}\right)\right|^{m^{\prime}} d x d t\right)^{\frac{1}{m^{\prime}}} .
\end{aligned}
$$

Hence

$$
\begin{aligned}
& \operatorname{ess} \sup _{0 \leq t \leq T} \int_{A_{k}(t)}\left|G_{k}\left(u_{n}(t)\right)\right|^{2} d x+2 \alpha \int_{0}^{T} \int_{A_{k}(t)} \frac{\left|D u_{n}\right|^{2}}{\left(1+\left|u_{n}\right|\right)^{\theta}} d x d t \\
& \leq 2\left\|f_{n}\right\|_{L^{m}(Q)}\left(\int_{0}^{T} \int_{A_{k}(t)}\left|G_{k}\left(u_{n}\right)\right|^{m^{\prime}} d x d t\right)^{\frac{1}{m^{\prime}}} .
\end{aligned}
$$


For all $1<\sigma<2,(2.10)$, Hölder's inequality and (2.3) imply that

$$
\begin{aligned}
& \int_{Q}\left|D G_{k}\left(u_{n}\right)\right|^{\sigma} d x d t=\int_{Q} \frac{\left|D G_{k}\left(u_{n}\right)\right|^{\sigma}}{\left(1+\left|u_{n}\right|\right)^{\theta \sigma / 2}}\left(1+\left|u_{n}\right|\right)^{\theta \sigma / 2} d x d t \\
& \leq\left(\int_{0}^{T} \int_{A_{k}(t)} \frac{\left|D G_{k}\left(u_{n}\right)\right|^{2}}{\left(1+\left|u_{n}\right|\right)^{\theta}} d x d t\right)^{\frac{\sigma}{2}}\left(\int_{0}^{T} \int_{A_{k}(t)}\left(1+\left|u_{n}\right|\right)^{\frac{\theta \sigma}{2-\sigma}} d x d t\right)^{\frac{2-\sigma}{2}} \\
& \leq \alpha^{-\frac{\sigma}{2}}\left\|f_{n}\right\|_{L^{m}(Q)}^{\frac{\sigma}{2}}\left(\int_{0}^{T} \int_{A_{k}(t)}\left|G_{k}\left(u_{n}\right)\right|^{m^{\prime}}\right)^{\frac{\sigma}{2 m^{\prime}}}\left(\int_{0}^{T} \int_{A_{k}(t)}\left(1+\left|u_{n}\right|\right)^{\frac{\theta \sigma}{2-\sigma}}\right)^{\frac{2-\sigma}{2}} \\
& \leq C_{2}\left(\int_{0}^{T} \int_{A_{k}(t)}\left|G_{k}\left(u_{n}\right)\right|^{m^{\prime}}\right)^{\frac{\sigma}{2 m^{\prime}}}\left(\int_{0}^{T} \int_{A_{k}(t)}\left(k+\left|G_{k}\left(u_{n}\right)\right|\right)^{\frac{\theta \sigma}{2-\sigma}}\right)^{\frac{2-\sigma}{2}} .
\end{aligned}
$$

The last term in the above inequality is due to $1+\left|u_{n}\right| \leq 2\left(k+\left|G_{k}\left(u_{n}\right)\right|\right)$ as $k \geq 1$. Let $\sigma=\frac{2 N+4-N \theta}{N+2}$, then we have $\frac{\theta \sigma}{2-\sigma}=\frac{(N+2) \sigma}{N}, 1<\sigma<2$ and $0<2-\sigma<1$. From (2.11), we get

$$
\begin{gathered}
\int_{Q}\left|D G_{k}\left(u_{n}\right)\right|^{\sigma} d x d t \leq 2^{\frac{\theta \sigma-2+\sigma}{2}} C_{2}\left(\int_{0}^{T} \int_{A_{k}(t)}\left|G_{k}\left(u_{n}\right)\right|^{m^{\prime}} d x d t\right)^{\frac{\sigma}{2 m^{\prime}}} \\
\cdot\left[k^{\frac{\theta \sigma}{2}}\left(\int_{0}^{T}\left|A_{k}(t)\right| d t\right)^{\frac{N \theta}{2(N+2)}}+\left(\int_{0}^{T} \int_{A_{k}(t)}\left|G_{k}\left(u_{n}\right)\right|^{\frac{(N+2) \sigma}{N}} d x d t\right)^{\frac{N \theta}{2(N+2)}}\right] .
\end{gathered}
$$

By Lemma 2.2(here $\left.v=G_{k}\left(u_{n}\right), h=\sigma, \varrho=2\right),(2.10)$ and (2.12), we obtain

$$
\begin{aligned}
& \int_{0}^{T} \int_{A_{k}(t)}\left|G_{k}\left(u_{n}\right)\right|^{\frac{(N+2) \sigma}{N}} d x d t \\
& \leq\left(\operatorname{ess} \sup _{0 \leq t \leq T} \int_{A_{k}(t)}\left|G_{k}\left(u_{n}(t)\right)\right|^{2} d x\right)^{\frac{\sigma}{N}} \int_{0}^{T} \int_{A_{k}(t)}\left|D G_{k}\left(u_{n}\right)\right|^{\sigma} d x d t \\
& \leq C_{3}\left(\int_{0}^{T} \int_{A_{k}(t)}\left|G_{k}\left(u_{n}\right)\right|^{m^{\prime}} d x d t\right)^{\frac{\sigma}{m^{\prime} N}}\left(\int_{0}^{T} \int_{A_{k}(t)}\left|G_{k}\left(u_{n}\right)\right|^{m^{\prime}} d x d t\right)^{\frac{\sigma}{2 m^{\prime}}} \\
& \cdot\left[k^{\frac{\theta \sigma}{2}}\left(\int_{0}^{T}\left|A_{k}(t)\right| d t\right)^{\frac{N \theta}{2(N+2)}}+\left(\int_{0}^{T} \int_{A_{k}(t)}\left|G_{k}\left(u_{n}\right)\right|^{\frac{(N+2) \sigma}{N}} d x d t\right)^{\frac{N \theta}{2(N+2)}}\right] \\
&=\left.C_{3}\left(\int_{0}^{T} \int_{A_{k}(t)}\left|G_{k}\left(u_{n}\right)\right|^{m^{\prime}} d x d t\right)^{\frac{\sigma(N+2)}{2 m^{\prime} N}}\right] \\
& \cdot\left[k^{\frac{\theta \sigma}{2}}\left(\int_{0}^{T}\left|A_{k}(t)\right| d t\right)^{\frac{N \theta}{2(N+2)}}+\left(\int_{0}^{T} \int_{A_{k}(t)}\left|G_{k}\left(u_{n}\right)\right|^{\frac{(N+2) \sigma}{N}} d x d t\right)^{\frac{N \theta}{2(N+2)}}\right] .
\end{aligned}
$$

By virtue of $m>\frac{N}{2}+1$ and $1<\sigma<2$, then we have $\frac{\sigma(N+2)}{m^{\prime} N}>1$. Using Hölder's inequality, we obtain

$$
\begin{aligned}
& \int_{0}^{T} \int_{A_{k}(t)}\left|G_{k}\left(u_{n}\right)\right|^{m^{\prime}} d x d t \\
& \leq\left(\int_{0}^{T} \int_{A_{k}(t)}\left|G_{k}\left(u_{n}\right)\right|^{\frac{(N+2) \sigma}{N}} d x d t\right)^{\frac{m^{\prime} N}{\sigma(N+2)}}\left(\int_{0}^{T}\left|A_{k}(t)\right| d t\right)^{1-\frac{m^{\prime} N}{\sigma(N+2)}} .
\end{aligned}
$$


(2.13) and (2.14) yield

$$
\begin{aligned}
& \int_{0}^{T} \int_{A_{k}(t)}\left|G_{k}\left(u_{n}\right)\right|^{\frac{(N+2) \sigma}{N}} d x d t \\
& \leq C_{3}\left(\int_{0}^{T} \int_{A_{k}(t)}\left|G_{k}\left(u_{n}\right)\right|^{\frac{(N+2) \sigma}{N}} d x d t\right)^{\frac{1}{2}}\left(\int_{0}^{T}\left|A_{k}(t)\right| d t\right)^{\frac{\sigma(N+2)(m-1)}{2 N m}-\frac{1}{2}} \\
& \quad \cdot\left[k^{\frac{\theta \sigma}{2}}\left(\int_{0}^{T}\left|A_{k}(t)\right| d t\right)^{\frac{N \theta}{2(N+2)}}+\left(\int_{0}^{T} \int_{A_{k}(t)}\left|G_{k}\left(u_{n}\right)\right|^{\frac{(N+2) \sigma}{N}} d x d t\right)^{\frac{N \theta}{2(N+2)}}\right] .
\end{aligned}
$$

Hence,

$$
\begin{aligned}
& \int_{0}^{T} \int_{A_{k}(t)}\left|G_{k}\left(u_{n}\right)\right|^{\frac{(N+2) \sigma}{N}} d x d t \\
& \leq C_{4}\left(\int_{0}^{T}\left|A_{k}(t)\right| d t\right)^{\frac{\sigma(N+2)(m-1)}{N m}-1}\left[k^{\theta \sigma}\left(\int_{0}^{T}\left|A_{k}(t)\right| d t\right)^{\frac{N \theta}{N+2}}\right. \\
& \left.\quad+\left(\int_{0}^{T} \int_{A_{k}(t)}\left|G_{k}\left(u_{n}\right)\right|^{\frac{(N+2) \sigma}{N}} d x d t\right)^{\frac{N \theta}{N+2}}\right]
\end{aligned}
$$

By virtue of $\theta<1+\frac{2}{N}$, then $\frac{N \theta}{N+2}<1$. Thus we can use Young's inequality with $\varepsilon$,

$$
\begin{aligned}
& C_{4}\left(\int_{0}^{T}\left|A_{k}(t)\right| d t\right)^{\frac{\sigma(N+2)(m-1)}{N m}-1}\left(\int_{0}^{T} \int_{A_{k}(t)}\left|G_{k}\left(u_{n}\right)\right|^{\frac{(N+2) \sigma}{N}} d x d t\right)^{\frac{N \theta}{N+2}} \\
& \leq \varepsilon \int_{0}^{T} \int_{A_{k}(t)}\left|G_{k}\left(u_{n}\right)\right|^{\frac{(N+2) \sigma}{N}} d x d t \\
& \quad+C(\varepsilon)\left(\int_{0}^{T}\left|A_{k}(t)\right| d t\right)^{\left[\frac{\sigma(N+2)(m-1)}{N m}-1\right] \frac{(N+2)}{N+2-N \theta}} .
\end{aligned}
$$

Applying (2.17) to (2.16) and taking $\varepsilon=\frac{1}{2}$, we get

$$
\begin{aligned}
\int_{0}^{T} \int_{A_{k}(t)}\left|G_{k}\left(u_{n}\right)\right|^{\frac{(N+2) \sigma}{N}} d x d t \leq & C_{5} k^{\theta \sigma}\left(\int_{0}^{T}\left|A_{k}(t)\right| d t\right)^{\frac{\sigma(N+2)(m-1)}{N m}-1+\frac{N \theta}{N+2}} \\
& +C_{5}\left(\int_{0}^{T}\left|A_{k}(t)\right| d t\right)^{\left[\frac{\sigma(N+2)(m-1)}{N m}-1\right] \frac{(N+2)}{N+2-N \theta}}
\end{aligned}
$$

The condition $m>\frac{N}{2}+1$ implies $\frac{\sigma(N+2)(m-1)}{N m}-1+\frac{N \theta}{N+2}>1$. Let $\lambda+1=\frac{\sigma(N+2)(m-1)}{N m}-$ $1+\frac{N \theta}{N+2}$, then $\lambda>0$. It's easy to see that $\left[\frac{\sigma(N+2)(m-1)}{N m}-1\right] \frac{(N+2)}{N+2-N \theta}>\frac{\sigma(N+2)(m-1)}{N m}-$ $1+\frac{N \theta}{N+2}=\lambda+1$, since $\sigma=\frac{2 N+4-N \theta}{N+2}$ and $m>\frac{N}{2}+1$. 
Hence, if $k \geq 1$, we have

$$
\begin{aligned}
& \int_{0}^{T} \int_{A_{k}(t)}\left|G_{k}\left(u_{n}\right)\right|^{\frac{(N+2) \sigma}{N}} d x d t \leq C_{5} k^{\theta \sigma}\left(\int_{0}^{T}\left|A_{k}(t)\right| d t\right)^{\lambda+1} \\
& \quad+C_{5}|Q|^{\left[\frac{\sigma(N+2)(m-1)}{N m}-1\right] \frac{(N+2)}{N+2-N \theta}-\lambda-1}\left(\int_{0}^{T}\left|A_{k}(t)\right| d t\right)^{\lambda+1} \\
& \leq C_{6} k^{\theta \sigma}\left(\int_{0}^{T}\left|A_{k}(t)\right| d t\right)^{\lambda+1},
\end{aligned}
$$

where $C_{6}=\max \left\{C_{5}, C_{5}|Q|^{\left[\frac{\sigma(N+2)(m-1)}{N m}-1\right] \frac{(N+2)}{N+2-N \theta}-\lambda-1}\right\}$. Recalling the property of $G_{k}(s)$, if $h>k$, we have $\left|G_{k}\left(u_{n}\right)\right|>h-k$ on $A_{h}(t)$, and $A_{h}(t) \subset A_{k}(t)$. Hence,

$$
\int_{0}^{T}\left|A_{h}(t)\right| d t \leq \frac{C_{6} k^{\theta \sigma}\left(\int_{0}^{T}\left|A_{k}(t)\right| d t\right)^{\lambda+1}}{(h-k)^{\frac{(N+2) \sigma}{N}}}, \quad \forall h>k \geq 1 .
$$

Let

$$
\varphi(k)=\int_{0}^{T}\left|A_{k}(t)\right| d t
$$

By virtue of $\theta \sigma=(2-\sigma) \frac{(N+2) \sigma}{N}, 2-\sigma<1,(2.20)$ can be written as

$$
\varphi(h) \leq \frac{C_{6} k^{(2-\sigma) \frac{(N+2) \sigma}{N}} \varphi(k)^{\lambda+1}}{(h-k)^{\frac{(N+2) \sigma}{N}}}, \quad \forall h>k \geq 1 .
$$

Thus we can use Lemma A.2 in [13] and obtain a positive constant $k^{*}$ independent of $n$ such that

$$
\varphi\left(k^{*}\right)=0 .
$$

Hence (2.23) yields (2.5).

Taking $u_{n}$ as a test function for problem $\left(P_{n}\right)$ and using $(2.5)$, it is easy to prove (2.6). In fact, we have

$$
\begin{aligned}
& \left.\frac{1}{2} \int_{\Omega} \mid u_{n}(T)\right)\left.\right|^{2} d x+\alpha \int_{Q} \frac{\left|D u_{n}\right|^{2}}{\left(1+\left|u_{n}\right|\right)^{\theta}} d x d t \\
& \left.\leq \frac{1}{2} \int_{\Omega} \mid u_{n}(T)\right)\left.\right|^{2} d x+\alpha \int_{Q} \frac{\left|D u_{n}\right|^{2}}{\left(1+\left|T_{n}\left(u_{n}\right)\right|\right)^{\theta}} d x d t \\
& \leq\left\|f_{n}\right\|_{L^{m}(Q)}\left(\int_{Q}\left|u_{n}\right|^{m^{\prime}} d x d t\right)^{\frac{1}{m^{\prime}}} .
\end{aligned}
$$

The above inequality and (2.5) yield

$$
\begin{aligned}
\int_{Q}\left|D u_{n}\right|^{2} d x d t & =\int_{Q} \frac{\left|D u_{n}\right|^{2}}{\left(1+\left|u_{n}\right|\right)^{\theta}}\left(1+\left|u_{n}\right|\right)^{\theta} d x d t \\
& \leq\left\|1+\left|u_{n}\right|\right\|_{L^{\infty}(Q)}^{\theta} \int_{Q} \frac{\left|D u_{n}\right|^{2}}{\left(1+\left|u_{n}\right|\right)^{\theta}} d x d t \\
& \leq\left(1+C_{1}\right)^{\theta} C_{1} \alpha^{-1}|Q|^{\frac{1}{m^{\prime}}}\left\|f_{n}\right\|_{L^{m}(Q)} .
\end{aligned}
$$

Thus by the above estimate and (2.3)-(2.5), we get (2.6). 
By the first equation of problem $\left(P_{n}\right)$ and combining with (2.3) and (2.6), we can get (2.7).

(ii) Suppose that $\theta=0$. Using the same arguments as the above proof, we only need to take $\theta=0$ in (2.10), $\theta=0, \sigma=2$ in (2.13)-(2.16) and (2.20) and delete the term $k^{(2-\sigma) \frac{(N+2) \sigma}{N}}$ in $(2.22)$.

Lemma 2.5. Assume that $m=\frac{N}{2}+1$ in (2.3)-(2.4) and (1.1)-(1.2) hold. Then for every solution $u_{n}$ of problem $\left(P_{n}\right)$, there exists a positive constant $C_{7}$ independent of $n$ such that

$$
\begin{aligned}
\left\|u_{n}\right\|_{L^{2}\left(0, T ; H_{0}^{1}(\Omega)\right)} & \leq C_{7}, \\
\left\|u_{n}\right\|_{L^{r}(Q)} & \leq C_{7}, \\
\left\|u_{n}^{\prime}\right\|_{L^{2}\left(0, T ; H^{-1}(\Omega)\right)+L^{m}(Q)} & \leq C_{7},
\end{aligned}
$$

for every $2 \leq r<+\infty$.

Proof. Let $\psi(s)=\left[(1+|s|)^{p}-1\right] \operatorname{sign}(s), \forall s \in R$, where $p>1$ is a positive constant which will be determined lately. For $\forall \tau \in(0, T]$, using $\psi\left(u_{n}(x, t)\right) \chi_{(0, \tau)}(t)$ as a test function for problem $\left(P_{n}\right)$, and combining with (1.1), we get

$$
\begin{aligned}
& \int_{\Omega} \Psi\left(u_{n}(x, \tau)\right) d x+p \alpha \int_{0}^{\tau} \int_{\Omega} \frac{\left|D u_{n}\right|^{2}}{\left(1+\left|u_{n}\right|\right)^{\theta}}\left(1+\left|u_{n}\right|\right)^{p-1} d x d t \\
& \leq \int_{0}^{\tau} \int_{\Omega}\left|f_{n}\right|\left[\left(1+\left|u_{n}\right|\right)^{p}-1\right] d x d t
\end{aligned}
$$

where $\Psi(s)=\int_{0}^{s} \psi(\xi) d \xi$.

By the definitions of $\psi(s)$ and $\Psi(s)$, we can get whenever $p>1$,

$$
\Psi(s) \geq \frac{1}{p+1}|s|^{p+1}, \quad \forall s \in R
$$

Thus (2.27)-(2.28) and Hölder's inequality imply that

$$
\begin{aligned}
& \frac{1}{p+1} \int_{\Omega}\left|u_{n}(x, \tau)\right|^{p+1} d x+p \alpha \int_{0}^{\tau} \int_{\Omega}\left|D u_{n}\right|^{2}\left(1+\left|u_{n}\right|\right)^{p-1-\theta} d x d t \\
& \leq\left\|f_{n}\right\|_{L^{m}(Q)}\left(\int_{Q}\left|\left(1+\left|u_{n}\right|\right)^{p}-1\right|^{m^{\prime}} d x d t\right)^{\frac{1}{m^{\prime}}} .
\end{aligned}
$$

If $p \geq 1+\theta$, the above estimate and (2.3) yield

$$
\begin{aligned}
& \text { ess } \sup _{0 \leq t \leq T} \int_{\Omega}\left[\left|u_{n}(x, t)\right|^{\frac{p+1-\theta}{2}}\right]^{\frac{2(p+1)}{p+1-\theta}} d x+\left.\left.\int_{Q}|D| u_{n}\right|^{\frac{p+1-\theta}{2}}\right|^{2} d x d t \\
& \leq C_{8}\left(\int_{Q}\left|u_{n}\right|^{p m^{\prime}} d x d t\right)^{\frac{1}{m^{\prime}}}+C_{8},
\end{aligned}
$$


where $C_{8}$ is a positive constant independent of $n$. By Lemma 2.2 (here $v(x, t)=$ $\left.\left|u_{n}(x, t)\right|^{\frac{p+1-\theta}{2}}, h=2, \varrho=\frac{2(p+1)}{p+1-\theta}\right)$, we obtain

$$
\begin{aligned}
& \int_{Q}\left[\left|u_{n}\right|^{\frac{p+1-\theta}{2}}\right]^{\frac{2\left(N+\frac{2(p+1)}{p+1-\theta}\right)}{N}} d x d t \\
& \leq\left.\left.\left(\operatorname{ess} \sup _{0 \leq t \leq T} \int_{\Omega}\left[\left|u_{n}(x, t)\right|^{\frac{p+1-\theta}{2}}\right]^{\frac{2(p+1)}{p+1-\theta}} d x\right)^{\frac{2}{N}} \int_{Q}|D| u_{n}\right|^{\frac{p+1-\theta}{2}}\right|^{2} d x d t \\
& \leq\left[C_{8}\left(\int_{Q}\left|u_{n}\right|^{p m^{\prime}} d x d t\right)^{\frac{1}{m^{\prime}}}+C_{8}\right]^{\frac{N+2}{N}} \leq C_{9}\left(\int_{Q}\left|u_{n}\right|^{p m^{\prime}} d x d t\right)^{\frac{N+2}{N m^{\prime}}}+C_{9} .
\end{aligned}
$$

That is

$$
\int_{Q}\left|u_{n}\right|^{\frac{2(p+1)+N(p+1-\theta)}{N}} d x d t \leq C_{9}\left(\int_{Q}\left|u_{n}\right|^{p m^{\prime}} d x d t\right)^{\frac{N+2}{N m^{\prime}}}+C_{9} .
$$

By virtue of $m=\frac{N}{2}+1, \theta<1+\frac{2}{N}$, we have

$$
p m^{\prime}<\frac{2(p+1)+N(p+1-\theta)}{N}, \quad \frac{N+2}{N m^{\prime}}=1, \quad \frac{p(N+2)}{2(p+1)+N(p+1-\theta)}<1 .
$$

Using Hölder's inequality and Young's inequality with $\varepsilon$, we obtain

$$
\begin{aligned}
& \left(\int_{Q}\left|u_{n}\right|^{p m^{\prime}} d x d t\right)^{\frac{N+2}{N m^{\prime}}} \\
& \leq\left(\int_{Q}\left|u_{n}\right|^{\frac{2(p+1)+N(p+1-\theta)}{N}} d x d t\right)^{\frac{p(N+2)}{2(p+1)+N(p+1-\theta)}}|Q|^{\left[1-\frac{N p m^{\prime}}{2(p+1)+N(p+1-\theta)}\right] \frac{(N+2)}{N m^{\prime}}} \\
& =\left(\int_{Q}\left|u_{n}\right|^{\frac{2(p+1)+N(p+1-\theta)}{N}} d x d t\right)^{\frac{p(N+2)}{2(p+1)+N(p+1-\theta)}}|Q|^{1-\frac{p(N+2)}{2(p+1)+N(p+1-\theta)}} \\
& \leq \varepsilon \int_{Q}\left|u_{n}\right|^{\frac{2(p+1)+N(p+1-\theta)}{N}} d x d t+C(\varepsilon) .
\end{aligned}
$$

Taking (2.33) in (2.32) and letting $\varepsilon=\frac{1}{2 C_{9}}$, we get

$$
\int_{Q}\left|u_{n}\right|^{\frac{2(p+1)+N(p+1-\theta)}{N}} d x d t \leq C_{10}
$$

Let

$$
\frac{2(p+1)+N(p+1-\theta)}{N}=r
$$

Then

$$
p=\frac{N r-2-N(1-\theta)}{N+2} .
$$

(2.34) and (2.35) yield (2.25).

To ensure $p \geq 1+\theta$, this needs $r \geq \frac{2(N+2)}{N}+\frac{2}{N} \theta$. Thus, if $r \geq \frac{2(N+2)}{N}+\frac{2}{N} \theta,(2.25)$ is proved. If $2 \leq r<\frac{2(N+2)}{N}+\frac{2}{N} \theta$, it is classical since $Q$ is bounded. 
By (2.3), (2.29) (here $\tau=T),(2.33)$ (here $\varepsilon=1),(2.34)$ and $p \geq 1+\theta$, we get

$$
\int_{Q}\left|D u_{n}\right|^{2} d x d t \leq \int_{Q}\left|D u_{n}\right|^{2}\left(1+\left|u_{n}\right|\right)^{p-1-\theta} d x d t \leq C_{11} .
$$

Thus (2.24) is proved. By (2.24) and (2.3), (2.26) can be obtained.

Lemma 2.6. Assume that $m$ in (2.3)-(2.4) satisfies (1.3), and (1.1) and (1.2) hold. Then for every solution $u_{n}$ of problem $\left(P_{n}\right)$, there exists a positive constant $C_{12}$ independent of $n$ such that

$$
\begin{aligned}
\left\|u_{n}\right\|_{L^{2}\left(0, T ; H_{0}^{1}(\Omega)\right)} & \leq C_{12}, \\
\left\|u_{n}\right\|_{L^{r}(Q)} & \leq C_{12}, \\
\left\|u_{n}^{\prime}\right\|_{L^{2}\left(0, T ; H^{-1}(\Omega)\right)+L^{m}(Q)} & \leq C_{12},
\end{aligned}
$$

where $r$ is defined in (1.4).

Proof. Here we simply revise the proof of Lemma 2.5. Let

$$
p m^{\prime}=\frac{2(p+1)+N(p+1-\theta)}{N} .
$$

Then

$$
p=\frac{[2+N(1-\theta)](m-1)}{N+2-2 m} .
$$

It is obvious to see that

$$
p m^{\prime}=r .
$$

By virtue of $m<\frac{N}{2}+1$, then we have $\frac{N+2}{N m^{\prime}}<1$. Using Young's inequality with $\varepsilon$, we obtain

$$
\left(\int_{Q}\left|u_{n}\right|^{p m^{\prime}} d x d t\right)^{\frac{N+2}{N m^{\prime}}} \leq \varepsilon \int_{Q}\left|u_{n}\right|^{p m^{\prime}} d x d t+C(\varepsilon) .
$$

Taking (2.44) in (2.32) and letting $\varepsilon=\frac{1}{2 C_{9}}$, by (2.41) and (2.43), we obtain (2.39). Furthermore, the condition $m \geq \frac{2(N+2+\theta)}{N+4-(N-2) \theta}$ in (1.3) ensures that $p \geq 1+\theta$ holds. The rest of the proof is the same as that of Lemma 2.4.

Lemma 2.7. Assume that $m$ in (2.3)-(2.4) satisfies (1.5) and $m>1$, and (1.1) and (1.2) hold. Then for every solution $u_{n}$ of problem $\left(P_{n}\right)$, there exists a positive constant $C_{13}$ independent of $n$ such that

$$
\begin{aligned}
\left\|u_{n}\right\|_{L^{q}\left(0, T ; W_{0}^{1, q}(\Omega)\right)} & \leq C_{13}, \\
\left\|u_{n}\right\|_{L^{r}(Q)} & \leq C_{13}, \\
\left\|u_{n}^{\prime}\right\|_{L^{q}\left(0, T ; W^{-1, q}(\Omega)\right)+L^{m}(Q)} & \leq C_{13},
\end{aligned}
$$

where $r$ and $q$ are defined in (1.6).

Proof. By the definitions of $\psi(s)$ and $\Psi(s)$ in the proof of Lemma 2.5, we also have if $0<p<1+\theta$,

$$
\Psi(s) \geq C_{p}|s|^{p+1}-\tilde{C}_{p}, \quad \forall s \in R
$$


where $C_{p}=\frac{1}{2(p+1)}, \tilde{C}_{p}=2^{\frac{1}{p}}, p$ will be fixed after. Replacing (2.28), (2.29) and (2.30) with (2.48) and the following two inequalities respectively,

$$
\begin{aligned}
& C_{p} \int_{\Omega}\left|u_{n}(x, \tau)\right|^{p+1} d x+p \alpha \int_{0}^{\tau} \int_{\Omega}\left|D u_{n}\right|^{2}\left(1+\left|u_{n}\right|\right)^{p-1-\theta} d x d t \\
& \leq\left\|f_{n}\right\|_{L^{m}(Q)}\left(\int_{Q}\left|\left(1+\left|u_{n}\right|\right)^{p}-1\right|^{m^{\prime}} d x d t\right)^{\frac{1}{m^{\prime}}}+\tilde{C}_{p}|\Omega|, \\
& \text { ess } \sup _{0 \leq t \leq T} \int_{\Omega}\left|u_{n}(x, t)\right|^{p+1} d x+\int_{Q} \frac{\left|D u_{n}\right|^{2}}{\left(1+\left|u_{n}\right|\right)^{\theta+1-p}} d x d t \\
& \leq C_{14}\left(\int_{Q}\left|u_{n}\right|^{p m^{\prime}} d x d t\right)^{\frac{1}{m^{\prime}}}+C_{14},
\end{aligned}
$$

where $C_{14}$ is a positive constant independent of $n$.

For all $q<2,(2.50)$ and Hölder's inequality imply that

$$
\begin{aligned}
& \int_{Q}\left|D u_{n}\right|^{q} d x d t=\int_{Q} \frac{\left|D u_{n}\right|^{q}}{\left(1+\left|u_{n}\right|\right)^{\frac{(\theta+1-p) q}{2}}}\left(1+\left|u_{n}\right|\right)^{\frac{(\theta+1-p) q}{2}} d x d t \\
& \leq\left(\int_{Q} \frac{\left|D u_{n}\right|^{2}}{\left(1+\left|u_{n}\right|\right)^{\theta+1-p}} d x d t\right)^{\frac{q}{2}}\left(\int_{Q}\left(1+\left|u_{n}\right|\right)^{\frac{(\theta+1-p) q}{2-q}} d x d t\right)^{\frac{2-q}{2}} \\
& \leq\left[C_{14}\left(\int_{Q}\left|u_{n}\right|^{p m^{\prime}} d x d t\right)^{\frac{1}{m^{\prime}}}+C_{14}\right]^{\frac{q}{2}} 2^{\frac{(\theta+1-p) q}{2}}\left[|Q|+\int_{Q}\left|u_{n}\right|^{\frac{(\theta+1-p) q}{2-q}} d x d t\right]^{\frac{2-q}{2}} \\
& \leq C_{15}\left[\left(\int_{Q}\left|u_{n}\right|^{p m^{\prime}} d x d t\right)^{\frac{1}{m^{\prime}}}+1\right]^{\frac{q}{2}}\left[\int_{Q}\left|u_{n}\right|^{\frac{(\theta+1-p) q}{2-q}} d x d t+1\right]^{\frac{2-q}{2}} .
\end{aligned}
$$

Let

$$
p m^{\prime}=\frac{(\theta+1-p) q}{2-q}
$$

It follows from (2.51) that

$$
\int_{Q}\left|D u_{n}\right|^{q} d x d t \leq C_{16}\left(\int_{Q}\left|u_{n}\right|^{p m^{\prime}} d x d t\right)^{\frac{q}{2 m^{\prime}}+\frac{2-q}{2}}+C_{16} .
$$

By Lemma 2.2 (here $\left.v(x, t)=u_{n}(x, t), h=q, \varrho=p+1\right)$, (2.50) and (2.53), we get

$$
\begin{aligned}
& \int_{Q}\left|u_{n}\right|^{\frac{(N+p+1) q}{N}} d x d t \leq\left(\text { ess } \sup _{0 \leq t \leq T} \int_{\Omega}\left|u_{n}(x, t)\right|^{p+1} d x\right)^{\frac{q}{N}} \int_{Q}\left|D u_{n}\right|^{q} d x d t \\
& \leq\left[C_{14}\left(\int_{Q}\left|u_{n}\right|^{p m^{\prime}} d x d t\right)^{\frac{1}{m^{\prime}}}+C_{14}\right]^{\frac{q}{N}}\left[C_{16}\left(\int_{Q}\left|u_{n}\right|^{p m^{\prime}} d x d t\right)^{\frac{q}{2 m^{\prime}}+\frac{2-q}{2}}+C_{16}\right] \\
& \leq C_{17}\left(\int_{Q}\left|u_{n}\right|^{p m^{\prime}} d x d t\right)^{\frac{q(N+2)}{2 N m^{\prime}}+\frac{2-q}{2}}+C_{17}
\end{aligned}
$$

Set

$$
p m^{\prime}=\frac{(p+1+N) q}{N} .
$$


Then this equality and (2.52) yield

$$
p=\frac{[2+N(1-\theta)](m-1)}{N+2-2 m}, \quad q=\frac{m[N(1-\theta)+2]}{N+1-(1+\theta)(m-1)} .
$$

Thus by direct calculation and the assumption of upper bound for $m$ in (1.5), we have

$$
p m^{\prime}=r, \quad p<1+\theta .
$$

Rewriting (2.54) as follows

$$
\int_{Q}\left|u_{n}\right|^{r} d x d t \leq C_{17}\left(\int_{Q}\left|u_{n}\right|^{r} d x d t\right)^{\frac{q(N+2)}{2 N m^{\prime}}+\frac{2-q}{2}}+C_{17} .
$$

By virtue of $m<\frac{N}{2}+1$, therefore we have $\frac{q(N+2)}{2 N m^{\prime}}+\frac{2-q}{2}<1$. Thus it follows from (2.57) and Young's inequality with $\varepsilon$ that (2.46). By (2.46), (2.53) and (2.56), we get

$$
\int_{Q}\left|D u_{n}\right|^{q} d x d t \leq C_{18}
$$

Thus (2.45) is proved. It is easy to obtain (2.47) from (2.45) and (2.3).

Lemma 2.8. Assume that $m$ in (2.3)-(2.4) satisfies (1.9), and (1.1) and (1.2) hold. Then for every solution $u_{n}$ of problem $\left(P_{n}\right)$, there exists a positive constant $C_{19}$ independent of $n$ and $k$ such that

$$
\begin{aligned}
\left\|u_{n}\right\|_{L^{\infty}\left(0, T ; L^{1}(\Omega)\right)} & \leq C_{19}, \\
\left\|T_{k}\left(u_{n}\right)\right\|_{L^{2}\left(0, T ; H_{0}^{1}(\Omega)\right)} & \leq C_{19}(1+k)^{\frac{1+\theta}{2}}, \\
\operatorname{meas}\left\{\left|u_{n}\right|>k\right\} & \leq \frac{C_{19}}{k^{r}}, \text { and } \\
\operatorname{meas}\left\{\left|D u_{n}\right|>k\right\} & \leq \frac{C_{19}}{k^{q}},
\end{aligned}
$$

where $r$ and $q$ as in (1.10) and (1.11).

Proof. The proof is divided into three cases.

(i) Suppose that $m>\frac{2(N+2)}{N+4}$. For all $\tau \in(0, T]$, choosing $T_{k}\left(u_{n}(x, t)\right) \chi_{(0, \tau)}(t)$ as a test function for problem $\left(P_{n}\right)$, and using (1.1) and Hölder's inequality, we get

$$
\begin{aligned}
& \int_{\Omega} S_{k}\left(u_{n}(x, \tau)\right) d x+\alpha \int_{0}^{\tau} \int_{\Omega} \frac{\left|D T_{k}\left(u_{n}\right)\right|^{2}}{\left(1+\left|u_{n}\right|\right)^{\theta}} d x d t \\
& \leq\left\|f_{n}\right\|_{L^{m}(Q)}\left(\int_{0}^{\tau} \int_{\Omega}\left|T_{k}\left(u_{n}\right)\right|^{m^{\prime}} d x d t\right)^{\frac{1}{m^{\prime}}} .
\end{aligned}
$$

By virtue of $S_{k}\left(u_{n}(x, \tau)\right) \geq \frac{\left|T_{k}\left(u_{n}(x, \tau)\right)\right|^{2}}{2}$, then we have

$$
\begin{aligned}
& \text { ess } \sup _{0 \leq t \leq T} \int_{\Omega}\left|T_{k}\left(u_{n}(x, t)\right)\right|^{2} d x+\int_{Q} \frac{\left|D T_{k}\left(u_{n}\right)\right|^{2}}{\left(1+\left|u_{n}\right|\right)^{\theta}} d x d t \\
& \leq C_{20}\left(\int_{Q}\left|T_{k}\left(u_{n}\right)\right|^{m^{\prime}} d x d t\right)^{\frac{1}{m^{\prime}}} .
\end{aligned}
$$


Hence

$$
\begin{aligned}
\int_{Q}\left|D T_{k}\left(u_{n}\right)\right|^{2} d x d t & =\int_{Q} \frac{\left|D T_{k}\left(u_{n}\right)\right|^{2}}{\left(1+\left|T_{k}\left(u_{n}\right)\right|\right)^{\theta}}\left(1+\left|T_{k}\left(u_{n}\right)\right|\right)^{\theta} d x d t \\
& \leq C_{20}(1+k)^{\theta}\left(\int_{Q}\left|T_{k}\left(u_{n}\right)\right|^{m^{\prime}} d x d t\right)^{\frac{1}{m^{\prime}}} .
\end{aligned}
$$

If $m>\frac{2(N+2)}{N+4}$, we have $m^{\prime}<\frac{2(N+2)}{N}$, thus we can choose $\rho<2$ such that $\frac{\rho(N+2)}{N}=m^{\prime}$. Then

$$
\rho=\frac{N m}{(N+2)(m-1)}
$$

For the above $\rho,(2.64)$ and Hölder's inequality imply that

$$
\begin{aligned}
& \int_{Q}\left|D T_{k}\left(u_{n}\right)\right|^{\rho} d x d t=\int_{Q} \frac{\left|D T_{k}\left(u_{n}\right)\right|^{\rho}}{\left(1+\left|T_{k}\left(u_{n}\right)\right|\right)^{\frac{\theta \rho}{2}}}\left(1+\left|T_{k}\left(u_{n}\right)\right|\right)^{\frac{\theta \rho}{2}} d x d t \\
& \leq\left(\int_{Q} \frac{\left|D T_{k}\left(u_{n}\right)\right|^{2}}{\left(1+\left|T_{k}\left(u_{n}\right)\right|\right)^{\theta}} d x d t\right)^{\frac{\rho}{2}}\left(\int_{Q}\left(1+\left|T_{k}\left(u_{n}\right)\right|\right)^{\frac{\theta \rho}{2-\rho}} d x d t\right)^{\frac{2-\rho}{2}} \\
& \leq C_{20}^{\frac{\rho}{2}}\left(\int_{Q}\left|T_{k}\left(u_{n}\right)\right|^{m^{\prime}} d x d t\right)^{\frac{\rho}{2 m^{\prime}}}\left(\int_{Q}\left(1+\left|T_{k}\left(u_{n}\right)\right|\right)^{\frac{\theta \rho}{2-\rho}} d x d t\right)^{\frac{2-\rho}{2}} .
\end{aligned}
$$

By Lemma 2.2 (here $v(x, t)=T_{k}\left(u_{n}(x, t)\right), h=\rho, \varrho=2$ ), we obtain

$$
\begin{gathered}
\int_{Q}\left|T_{k}\left(u_{n}\right)\right|^{\frac{(N+2) \rho}{N}} d x d t \leq\left(\operatorname{ess} \sup _{0 \leq t \leq T} \int_{\Omega}\left|T_{k}\left(u_{n}\right)\right|^{2} d x\right)^{\frac{\rho}{N}} \int_{Q}\left|D T_{k}\left(u_{n}\right)\right|^{\rho} d x d t \\
\leq C_{20}^{\frac{\rho}{N}}\left(\int_{Q}\left|T_{k}\left(u_{n}\right)\right|^{m^{\prime}} d x d t\right)^{\frac{\rho}{N m^{\prime}}} C_{20}^{\frac{\rho}{2}}\left(\int_{Q}\left|T_{k}\left(u_{n}\right)\right|^{m^{\prime}} d x d t\right)^{\frac{\rho}{2 m^{\prime}}} \\
\cdot\left(\int_{Q}\left(1+\left|T_{k}\left(u_{n}\right)\right|\right)^{\frac{\theta \rho}{2-\rho}} d x d t\right)^{\frac{2-\rho}{2}} \\
=C_{21}\left(\int_{Q}\left|T_{k}\left(u_{n}\right)\right|^{m^{\prime}} d x d t\right)^{\frac{\rho(N+2)}{2 N m^{\prime}}}\left(\int_{Q}\left(1+\left|T_{k}\left(u_{n}\right)\right|\right)^{\frac{\theta \rho}{2-\rho}} d x d t\right)^{\frac{2-\rho}{2}} .
\end{gathered}
$$

Now $m>\frac{2(N+2)}{N+4}$ and (1.9) imply $m \leq \frac{N+2+\theta}{N+3-\theta(N-1)}$. However, by virtue of $\theta<1+\frac{2}{N}$, then

$$
\frac{N+2+\theta}{N+3-\theta(N-1)}<\frac{2(N+2)-N \theta}{N+4-N \theta}
$$


Thus from the above inequality and (2.66), we can deduce that $\frac{\theta \rho}{2-\rho}>m^{\prime}$, if $k \geq 1$, (2.68) yields

$$
\begin{aligned}
\int_{Q}\left|T_{k}\left(u_{n}\right)\right|^{\frac{(N+2) \rho}{N}} d x d t=\int_{Q}\left|T_{k}\left(u_{n}\right)\right|^{m^{\prime}} d x d t \\
=C_{21}\left(\int_{Q}\left|T_{k}\left(u_{n}\right)\right|^{m^{\prime}} d x d t\right)^{\frac{\rho(N+2)}{2 N m^{\prime}}} \\
\cdot\left(\int_{Q}\left(1+\left|T_{k}\left(u_{n}\right)\right|\right)^{\frac{\theta \rho}{2-\rho}-m^{\prime}}\left(1+\left|T_{k}\left(u_{n}\right)\right|\right)^{m^{\prime}} d x d t\right)^{\frac{2-\rho}{2}} \\
\leq C_{21}\left(\int_{Q}\left|T_{k}\left(u_{n}\right)\right|^{m^{\prime}} d x d t\right)^{\frac{\rho(N+2)}{2 N m^{\prime}}} \\
\cdot(2 k)^{\left(\frac{\theta \rho}{2-\rho}-m^{\prime}\right) \frac{(2-\rho)}{2}}\left(\int_{Q}\left(1+\left|T_{k}\left(u_{n}\right)\right|\right)^{m^{\prime}} d x d t\right)^{\frac{2-\rho}{2}} \\
\leq C_{21}\left(\int_{Q}\left|T_{k}\left(u_{n}\right)\right|^{m^{\prime}} d x d t\right)^{\frac{\rho(N+2)}{2 N m^{\prime}}} \\
\quad \cdot(2 k)^{\left(\frac{\theta \rho}{2-\rho}-m^{\prime}\right) \frac{(2-\rho)}{2}}\left(2^{m^{\prime}}|Q|+2^{m^{\prime}} \int_{Q}\left|T_{k}\left(u_{n}\right)\right|^{m^{\prime}} d x d t\right)^{\frac{2-\rho}{2}} \\
\leq C_{22} k^{\frac{\theta \rho}{2}-\frac{(2-\rho) m^{\prime}}{2}}\left(\int_{Q}\left|T_{k}\left(u_{n}\right)\right|^{m^{\prime}} d x d t\right)^{\frac{\rho(N+2)}{2 N m^{\prime}}}\left(1+\int_{Q}\left|T_{k}\left(u_{n}\right)\right|^{m^{\prime}} d x d t\right)^{\frac{2-\rho}{2}},
\end{aligned}
$$

where $C_{22}=2^{\frac{\theta \rho}{2}} C_{21}(|Q|+1)^{\frac{2-\rho}{2}}$.

If $\int_{Q}\left|T_{k}\left(u_{n}\right)\right|^{m^{\prime}} d x d t \geq 1$, it follows from (2.69) that

$$
\int_{Q}\left|T_{k}\left(u_{n}\right)\right|^{m^{\prime}} d x d t \leq C_{22} 2^{\frac{2-\rho}{2}} k^{\frac{\theta \rho}{2}-\frac{(2-\rho) m^{\prime}}{2}}\left(\int_{Q}\left|T_{k}\left(u_{n}\right)\right|^{m^{\prime}} d x d t\right)^{\frac{\rho(N+2)}{2 N m^{\prime}}+\frac{2-\rho}{2}} .
$$

Hence

$$
\left(\int_{Q}\left|T_{k}\left(u_{n}\right)\right|^{m^{\prime}} d x d t\right)^{1-\frac{\rho(N+2)}{2 N m^{\prime}}-\frac{2-\rho}{2}} \leq C_{22} 2^{\frac{2-\rho}{2}} k^{\frac{\theta \rho}{2}-\frac{(2-\rho) m^{\prime}}{2}} .
$$

Thus we get

$$
\begin{aligned}
\int_{Q}\left|T_{k}\left(u_{n}\right)\right|^{m^{\prime}} d x d t & \leq\left(C_{22} 2^{\frac{2-\rho}{2}}\right)^{\frac{1}{1-\frac{\rho(N+2)}{2 N m^{\prime}}-\frac{2-\rho}{2}}} k^{\left[\frac{\theta \rho}{2}-\frac{(2-\rho) m^{\prime}}{2}\right] \frac{1}{1-\frac{\rho(N+2)}{2 N m^{\prime}}-\frac{2-\rho}{2}}} \\
& =C_{23} k^{\frac{\left[\theta \rho-(2-\rho) m^{\prime}\right] N m^{\prime}}{N m^{\prime} \rho-(N+2) \rho}} \\
& =C_{23} k^{-\frac{m[N(m-2)+4(m-1)]-\theta N m(m-1)}{(m-1)(N-2 m+2)}}
\end{aligned}
$$

where $C_{23}=\left(C_{22} 2^{\frac{2-\rho}{2}}\right)^{\frac{1}{1-\frac{\rho(N+2)}{2 N m^{\prime}}-\frac{2-\rho}{2}}}=\left(C_{22} 2^{\frac{2-\rho}{2}}\right)^{\frac{2 N m^{\prime}}{N m^{\prime}-\rho(N+2)}}$. The above second equality is due to $\rho=\frac{N m}{(N+2)(m-1)}$. Now $\theta<1+\frac{2}{N}$ and $m \leq \frac{N+2+\theta}{N+3-\theta(N-1)}$ imply $N-2 m+2>0$. Moreover, $\frac{N+2+\theta}{N+3-\theta(N-1)}<\frac{2(N+2)-N \theta}{N+4-N \theta}$ yields $N(m-2)+4(m-1)-\theta N(m-1)<0$. 
Thus we get

$$
-\frac{m[N(m-2)+4(m-1)]-\theta N m(m-1)}{(m-1)(N-2 m+2)}>0 .
$$

If $\int_{Q}\left|T_{k}\left(u_{n}\right)\right|^{m^{\prime}} d x d t \leq 1$, by virtue of $k \geq 1$, then

$$
\int_{Q}\left|T_{k}\left(u_{n}\right)\right|^{m^{\prime}} d x d t \leq 1 \leq k^{-\frac{m[N(m-2)+4(m-1)]-\theta N m(m-1)}{(m-1)(N-2 m+2)}} .
$$

Let $C_{24}=\max \left\{C_{23}, 1\right\}$. It is obvious to see that for any $k \geq 1$,

$$
\int_{Q}\left|T_{k}\left(u_{n}\right)\right|^{m^{\prime}} d x d t \leq C_{24} k^{-\frac{m[N(m-2)+4(m-1)]-\theta N m(m-1)}{(m-1)(N-2 m+2)}} .
$$

Because $m^{\prime}>-\frac{m[N(m-2)+4(m-1)]-\theta N m(m-1)}{(m-1)(N-2 m+2)}$ which is due to $\theta<1+\frac{2}{N}$, if $k \leq 1$, we have

$$
\int_{Q}\left|T_{k}\left(u_{n}\right)\right|^{m^{\prime}} d x d t \leq|Q| k^{m^{\prime}} \leq|Q| k^{-\frac{m[N(m-2)+4(m-1)]-\theta N m(m-1)}{(m-1)(N-2 m+2)}} .
$$

Let $C_{25}=\max \left\{C_{24},|Q|\right\}$. It follows from (2.71) and (2.72) that for any $k>0$,

$$
\int_{Q}\left|T_{k}\left(u_{n}\right)\right|^{m^{\prime}} d x d t \leq C_{25} k^{-\frac{m[N(m-2)+4(m-1)]-\theta N m(m-1)}{(m-1)(N-2 m+2)}} .
$$

Therefore we have

$$
k^{m^{\prime}} \operatorname{meas}\left\{(x, t) \in Q:\left|u_{n}(x, t)\right|>k\right\} \leq C_{25} k^{-\frac{m[N(m-2)+4(m-1)]-\theta N m(m-1)}{(m-1)(N-2 m+2)}} .
$$

Namely,

$$
\begin{aligned}
\operatorname{meas}\left\{(x, t) \in Q:\left|u_{n}(x, t)\right|>k\right\} & \leq C_{25} k^{-\frac{m[N(m-2)+4(m-1)]-\theta N m(m-1)}{(m-1)(N-2 m+2)}-m^{\prime}} \\
& =C_{25} k^{-\frac{m[N(1-\theta)+2]}{N+2-2 m}}=C_{25} k^{-r} .
\end{aligned}
$$

Thus (2.61) is proved.

Now (2.65) and (2.73) yield

$$
\int_{Q}\left|D T_{k}\left(u_{n}\right)\right|^{2} d x d t \leq C_{26}(1+k)^{\theta} k^{-\frac{[N(m-2)+4(m-1)]-\theta N(m-1)}{(N-2 m+2)}} .
$$

By (2.76) and Lemma 2.3 (here $v(x, t)=u_{n}(x, t), \quad \mu=\frac{m[N(1-\theta)+2]}{N+2-2 m}, \gamma=\theta, \quad \nu=$ $\left.\frac{-[N(m-2)+4(m-1)]+\theta(N m-2 m+2)}{N-2 m+2}, \delta=q\right)$, we can obtain (2.62).

(ii) Suppose that $1<m \leq \frac{2(N+2)}{N+4}$. Note that $m^{\prime} \geq \frac{2(N+2)}{N}$. Then we have

$$
\left(\int_{Q}\left|T_{k}\left(u_{n}\right)\right|^{m^{\prime}} d x d t\right)^{\frac{1}{m^{\prime}}} \leq k^{1-\frac{2(N+2)}{N m^{\prime}}}\left(\int_{Q}\left|T_{k}\left(u_{n}\right)\right|^{\frac{2(N+2)}{N}} d x d t\right)^{\frac{1}{m^{\prime}}} .
$$

From (2.64)-(2.65) and (2.77), we get

$$
\begin{gathered}
\operatorname{ess} \sup _{0 \leq t \leq T} \int_{\Omega}\left|T_{k}\left(u_{n}(x, t)\right)\right|^{2} d x \leq C_{20} k^{1-\frac{2(N+2)}{N m^{\prime}}}\left(\int_{Q}\left|T_{k}\left(u_{n}\right)\right|^{\frac{2(N+2)}{N}} d x d t\right)^{\frac{1}{m^{\prime}}}, \\
\int_{Q}\left|D T_{k}\left(u_{n}\right)\right|^{2} d x d t \leq C_{20}(1+k)^{\theta} k^{1-\frac{2(N+2)}{N m^{\prime}}}\left(\int_{Q}\left|T_{k}\left(u_{n}\right)\right|^{\frac{2(N+2)}{N}} d x d t\right)^{\frac{1}{m^{\prime}}} .
\end{gathered}
$$


By (2.78)-(2.79) and Lemma 2.2 (here $\left.v(x, t)=T_{k}\left(u_{n}(x, t)\right), h=2, \varrho=2\right)$, we conclude

$$
\int_{Q}\left|T_{k}\left(u_{n}\right)\right|^{\frac{2(N+2)}{N}} d x d t \leq C_{27} k^{\frac{(N+2)(-N m+2 N-4 m+4)+\theta N^{2} m}{N(N-2 m+2)}}
$$

Indeed,

$$
\begin{aligned}
& \int_{Q}\left|T_{k}\left(u_{n}\right)\right|^{\frac{2(N+2)}{N}} d x d t \leq\left(\operatorname{ess} \sup _{0 \leq t \leq T} \int_{\Omega}\left|T_{k}\left(u_{n}\right)\right|^{2} d x\right)^{\frac{2}{N}} \int_{Q}\left|D T_{k}\left(u_{n}\right)\right|^{2} d x d t \\
& \leq C_{20}^{\frac{2}{N}+1}(1+k)^{\theta} k^{\left[1-\frac{2(N+2)}{N m^{\prime}}\right]\left(\frac{2}{N}+1\right)}\left(\int_{Q}\left|T_{k}\left(u_{n}\right)\right|^{\frac{2(N+2)}{N}} d x d t\right)^{\frac{N+2}{N m^{\prime}}} .
\end{aligned}
$$

By virtue of $m \leq \frac{2(N+2)}{N+4}$, then $\frac{N+2}{N m^{\prime}} \leq \frac{1}{2}$. Thus we get

$$
\left(\int_{Q}\left|T_{k}\left(u_{n}\right)\right|^{\frac{2(N+2)}{N}} d x d t\right)^{1-\frac{N+2}{N m^{\prime}}} \leq C_{20}^{\frac{2}{N}+1}(1+k)^{\theta} k^{\left[1-\frac{2(N+2)}{N m^{\prime}}\right]\left(\frac{2}{N}+1\right)} .
$$

Hence

$$
\begin{aligned}
\int_{Q}\left|T_{k}\left(u_{n}\right)\right|^{\frac{2(N+2)}{N}} d x d t & \leq\left(C_{20}^{\frac{2}{N}+1}(1+k)^{\theta} k^{\left[1-\frac{2(N+2)}{N m^{\prime}}\right]\left(\frac{2}{N}+1\right)}\right)^{\frac{1}{1-\frac{N+2}{N m^{\prime}}}} \\
& =C_{20}^{\frac{(N+2) m}{N-2 m+2}}(1+k)^{\frac{N m \theta}{N-2 m+2}} k^{\frac{(N+2)(-N m+2 N-4 m+4)}{N(N-2 m+2)}} .
\end{aligned}
$$

If $k \geq 1$, it follows from $(2.82)$ that

$$
\int_{Q}\left|T_{k}\left(u_{n}\right)\right|^{\frac{2(N+2)}{N}} d x d t \leq 2^{\frac{N m \theta}{N-2 m+2}} C_{20}^{\frac{(N+2) m}{N-2 m+2}} k^{\frac{(N+2)(-N m+2 N-4 m+4)+\theta N^{2} m}{N(N-2 m+2)}} .
$$

If $k \leq 1$, since $\frac{2(N+2)}{N}>\frac{(N+2)(-N m+2 N-4 m+4)+\theta N^{2} m}{N(N-2 m+2)}$ which is due to $\theta<1+\frac{2}{N}$, we have

$$
\int_{Q}\left|T_{k}\left(u_{n}\right)\right|^{\frac{2(N+2)}{N}} d x d t \leq|Q| k^{\frac{2(N+2)}{N}} \leq|Q| k^{\frac{(N+2)(-N m+2 N-4 m+4)+\theta N^{2} m}{N(N-2 m+2)}} .
$$

Thus (2.83) and (2.84) yield (2.80). Therefore from (2.80) we can obtain (2.61). Finally, (2.62) can be deduced from (2.79)-(2.80) and Lemma 2.3.

(iii) Suppose that $m=1$. We only need to replace $\left(\int_{Q}\left|T_{k}\left(u_{n}\right)\right|^{m^{\prime}} d x d t\right)^{\frac{1}{m^{\prime}}}$ with $|Q|^{\frac{1}{m^{\prime}}} k$ in $(2.63)-(2.65)$. That is

$$
\begin{aligned}
& \int_{\Omega} S_{k}\left(u_{n}(x, \tau)\right) d x+\alpha \int_{0}^{\tau} \int_{\Omega} \frac{\left|D T_{k}\left(u_{n}\right)\right|^{2}}{\left(1+\left|u_{n}\right|\right)^{\theta}} d x d t \leq\left\|f_{n}\right\|_{L^{m}(Q)}|Q|^{\frac{1}{m^{\prime}}} k, \\
& \text { ess } \sup _{0 \leq t \leq T} \int_{\Omega}\left|T_{k}\left(u_{n}(x, t)\right)\right|^{2} d x+\int_{Q} \frac{\left|D T_{k}\left(u_{n}\right)\right|^{2}}{\left(1+\left|u_{n}\right|\right)^{\theta}} d x d t \leq C_{20}|Q|^{\frac{1}{m^{\prime}}} k, \\
& \int_{Q}\left|D T_{k}\left(u_{n}\right)\right|^{2} d x d t=\int_{Q} \frac{\left|D T_{k}\left(u_{n}\right)\right|^{2}}{\left(1+\left|T_{k}\left(u_{n}\right)\right|\right)^{\theta}}\left(1+\left|T_{k}\left(u_{n}\right)\right|\right)^{\theta} d x d t \\
& \leq C_{20}|Q|^{\frac{1}{m^{\prime}}}(1+k)^{\theta} k \text {. }
\end{aligned}
$$


By (2.86)-(2.87) and Lemma $2.2\left(\right.$ here $\left.v(x, t)=T_{k}\left(u_{n}(x, t)\right), h=2, \varrho=2\right)$, going through the same process as that of $(2.80)$, we obtain

$$
\int_{Q}\left|T_{k}\left(u_{n}\right)\right|^{\frac{2(N+2)}{N}} d x d t \leq C_{28} k^{\frac{N+2+\theta N}{N}} .
$$

Thus it's easy to get (2.61) by (2.88). Now (2.87)-(2.88) and Lemma 2.3 imply that (2.62) holds.

Taking $T_{1}\left(u_{n}\right) \chi_{(0, \tau)}(t)$ as a test function for problem $\left(P_{n}\right)$, and using (1.1) and Hölder's inequality, we get

$$
\begin{aligned}
& \int_{\Omega} S_{1}\left(u_{n}(x, \tau)\right) d x+\alpha \int_{0}^{\tau} \int_{\Omega} \frac{\left|D T_{1}\left(u_{n}\right)\right|^{2}}{\left(1+\left|u_{n}\right|\right)^{\theta}} d x d t \\
& \leq\left\|f_{n}\right\|_{L^{m}(Q)}\left(\int_{0}^{\tau} \int_{\Omega}\left|T_{1}\left(u_{n}\right)\right|^{m^{\prime}} d x d t\right)^{\frac{1}{m^{\prime}}} .
\end{aligned}
$$

Note that $|s|-1 / 2 \leq S_{1}(s) \leq|s|$, for any $s \in R$. Then we have

$$
\operatorname{ess} \sup _{0 \leq t \leq T} \int_{\Omega}\left|u_{n}(x, t)\right| d x \leq\left\|f_{n}\right\|_{L^{m}(Q)}|Q|^{\frac{1}{m^{\prime}}}+\frac{1}{2}|\Omega| .
$$

So (2.3) and (2.90) yield (2.59).

By (2.86) and (2.87), we obtain

$$
\begin{aligned}
\int_{Q}\left|T_{k}\left(u_{n}(x, t)\right)\right|^{2} d x d t & \leq C_{20}|Q|^{\frac{1}{m^{\prime}}} T k \\
\int_{Q}\left|D T_{k}\left(u_{n}\right)\right|^{2} d x d t & \leq C_{20}|Q|^{\frac{1}{m^{\prime}}}(1+k)^{\theta} k .
\end{aligned}
$$

The above two inequalities imply (2.60).

Lemma 2.9. Assume that $f_{n} \equiv 0, u_{0} \in L^{d}(\Omega)$ in problem $\left(P_{n}\right)$, where $1 \leq d \leq$ $d_{0}, d_{0}$ as in Theorem 1.6, and (1.1) holds. Then for every solution $u_{n}$ of problem $\left(P_{n}\right)$, there exists a positive constant $C_{29}$ independent of $n$ and $k$ such that

$$
\begin{aligned}
\left\|u_{n}\right\|_{L^{\infty}\left(0, T ; L^{d}(\Omega)\right)} & \leq C_{29}, \\
\left\|T_{k}\left(u_{n}\right)\right\|_{L^{2}\left(0, T ; H_{0}^{1}(\Omega)\right)} & \leq C_{29}(1+k)^{\frac{\theta-d+2}{2}} .
\end{aligned}
$$

(i) Suppose that $0 \leq \theta \leq \frac{2}{N-1}$. If $1 \leq d \leq \frac{N \theta}{2}$, then

$$
\operatorname{meas}\left\{\left|D u_{n}\right|>k\right\} \leq \frac{C_{29}}{k^{q_{1}}},
$$

where $q_{1}=\frac{2 d}{\theta+2}$.

If $\max \left\{1, \frac{N \theta}{2}\right\} \leq d \leq d_{0}$, then

$$
\operatorname{meas}\left\{\left|D u_{n}\right|>k\right\} \leq \frac{C_{29}}{k^{q_{2}}}
$$

where $q_{2}=\frac{(N+2) d-N \theta}{N+d}$.

(ii) Suppose that $\frac{2}{N-1} \leq \theta$. If $1 \leq d \leq d_{0}$, then

$$
\operatorname{meas}\left\{\left|D u_{n}\right|>k\right\} \leq \frac{C_{29}}{k^{q_{1}}},
$$

where $q_{1}=\frac{2 d}{\theta+2}$. 
Proof. Using $\left[\left(1+\left|u_{n}\right|\right)^{d-1}-1\right] \operatorname{sign}\left(u_{n}\right)$ as a test function for problem $\left(P_{n}\right)$, we can obtain

$$
\operatorname{ess} \sup _{0 \leq t \leq T} \int_{\Omega}\left(1+\left|u_{n}\right|\right)^{d} d x+\int_{Q} \frac{\left|D u_{n}\right|^{2}}{\left(1+\left|u_{n}\right|\right)^{\theta-d+2}} d x d t \leq C_{30} .
$$

Thus the above estimate yields (2.93). Hence for any $k>0$,

$$
\begin{aligned}
\int_{Q}\left|D T_{k}\left(u_{n}\right)\right|^{2} d x d t & =\int_{Q} \frac{\left|D T_{k}\left(u_{n}\right)\right|^{2}}{\left(1+\left|T_{k}\left(u_{n}\right)\right|\right)^{\theta-d+2}}\left(1+\left|T_{k}\left(u_{n}\right)\right|\right)^{\theta-d+2} d x d t \\
& \leq C_{30}(1+k)^{\theta-d+2} .
\end{aligned}
$$

From (2.99) we get (2.94). Now (2.98) yields

$$
\int_{Q}\left|T_{k}\left(u_{n}\right)\right|^{d} d x \leq T C_{30} .
$$

The proof is divided into three cases:

(i) Suppose that $0 \leq \theta \leq \frac{2}{N-1}$ whenever $1 \leq d \leq \frac{N \theta}{2}$. Now (2.100) implies that

$$
\operatorname{meas}\left\{\left|u_{n}\right|>k\right\} \leq \frac{T C_{30}}{k^{d}}
$$

From (2.99), we deduce for any $k \geq 1, \lambda \geq 1$,

$$
\operatorname{meas}\left\{\left|D u_{n}\right|>\lambda,\left|u_{n}\right| \leq k\right\} \leq \frac{C_{30} 2^{\theta-d+2} k^{\theta-d+2}}{\lambda^{2}} .
$$

Hence

$$
\begin{aligned}
\operatorname{meas}\left\{\left|D u_{n}\right|>\lambda\right\} & \leq \operatorname{meas}\left\{\left|D u_{n}\right|>\lambda,\left|u_{n}\right| \leq k\right\}+\operatorname{meas}\left\{\left|u_{n}\right|>k\right\} \\
& \leq \frac{C_{30} 2^{\theta-d+2} k^{\theta-d+2}}{\lambda^{2}}+\frac{T C_{30}}{k^{d}} .
\end{aligned}
$$

Choosing $k=\lambda^{\frac{2}{\theta+2}}$, we obtain

$$
\operatorname{meas}\left\{\left|D u_{n}\right|>\lambda\right\} \leq \frac{C_{31}}{\lambda^{\frac{2 d}{\theta+2}}}
$$

As $\lambda<1$, by virtue of

$$
\operatorname{meas}\left\{\left|D u_{n}\right|>\lambda\right\} \leq|Q| \leq \frac{|Q|}{\lambda^{\frac{2 d}{\theta+2}}} .
$$

Thus let $C_{29}=\max \left\{|Q|, C_{31}\right\}$ and replace $\lambda$ by $k$, the above inequalities imply (2.95).

(ii) Suppose that $0<\theta \leq \frac{2}{N-1}$ whenever $\max \left\{1, \frac{N \theta}{2}\right\} \leq d \leq d_{0}$. By (2.98)-(2.99) and Lemma 2.2 (here $v(x, t)=T_{k}\left(u_{n}(x, t)\right), h=2, \varrho=d$ ), we conclude

$$
\begin{aligned}
\int_{Q}\left|T_{k}\left(u_{n}\right)\right|^{\frac{2(N+d)}{N}} d x d t & \leq\left(\operatorname{ess} \sup _{0 \leq t \leq T} \int_{\Omega}\left|T_{k}\left(u_{n}\right)\right|^{d} d x\right)^{\frac{2}{N}} \int_{Q}\left|D T_{k}\left(u_{n}\right)\right|^{2} d x d t \\
& \leq C_{31}(1+k)^{\theta-d+2}
\end{aligned}
$$

Thus we get for any $k>0$,

$$
\operatorname{meas}\left\{\left|u_{n}\right|>k\right\} \leq \frac{\left(|Q|+C_{31} 2^{\theta-d+2}\right)}{k^{\frac{(N+2) d-N \theta}{N}}} .
$$

The proceeding is the same as that of (i), here we only replace (2.101) by (2.107). 
(iii) Suppose that $\frac{2}{N-1} \leq \theta$ whenever $1 \leq d \leq d_{0}$. The proof is the same as that of (i) because $d_{0} \leq \frac{N \theta}{2}$ in the case.

\section{Proof of Theorems 1.1-1.6}

Because the proofs of Theorem 1.1, Theorem 1.2 and Theorem 1.3 are similar to that of Theorem 1.4, the proof of Theorem 1.6 is also similar to that of Theorem 1.5, here we only give the proof of Theorem 1.4 and Theorem 1.5.

Proof of Theorem 1.4. By Lemma 2.7, there exist a subsequence of $\left\{u_{n}\right\}$ (still denoted by $\left.\left\{u_{n}\right\}\right)$ and a measurable function $u$ such that

$$
\begin{array}{ll}
u_{n} \longrightarrow u & \text { weakly in } L^{q}\left(0, T ; W_{0}^{1, q}(\Omega)\right), \\
u_{n}^{\prime} \longrightarrow u^{\prime} & \text { weakly in } L^{q}\left(0, T ; W^{-1, q}(\Omega)\right)+L^{m}(Q), \text { and } \\
u_{n} \longrightarrow u & \text { weakly in } L^{r}(Q) .
\end{array}
$$

Now (3.1)-(3.2) and a compactness result (see [27]) imply that

$$
u_{n} \longrightarrow u \text { strongly in } L^{1}(Q) \text {. }
$$

Hence

$$
u_{n} \longrightarrow u \text { a.e. in } \mathrm{Q},
$$

so (1.1) and (3.5) yield

$$
a\left(x, t, T_{n}\left(u_{n}\right)\right) \longrightarrow a(x, t, u) \quad \text { weak }^{*} \text { in } L^{\infty}(Q) .
$$

For any given $\psi$ in $C^{\infty}(\bar{Q})$ which is zero in a neighborhood of $\partial \Omega \times(0, T)$ and $\Omega \times\{T\}$, using $\psi$ as a test function for problem $\left(P_{n}\right)$, we have

$$
-\int_{Q} u_{n} \psi^{\prime} d x d t+\int_{Q} a\left(x, t, T_{n}\left(u_{n}\right)\right) D u_{n} D \psi d x d t=\int_{Q} f_{n} \psi d x d t .
$$

Let $n \rightarrow \infty$, by (2.4), (3.1), (3.4) and (3.6), we get

$$
-\int_{Q} u \psi^{\prime} d x d t+\int_{Q} a(x, t, u) D u D \psi d x d t=\int_{Q} f \psi d x d t .
$$

Here we only give the detailed proof of the second term on the left side of (3.8), the other terms are easily got.

By the absolute continuity of the integral, for all $\varepsilon>0$, there exists $\delta>0$ such that for every measurable subset $E \subset Q$ of measure less than $\delta$,

$$
\left(\int_{E}|D \psi|^{q^{\prime}} d x d t\right)^{\frac{1}{q^{\prime}}}<\frac{\varepsilon}{6 \beta C_{13}},
$$

where $\beta$ is as in (1.1), $C_{13}$ is a positive constant defined in Lemma 2.7.

Now (3.5) yields

$$
a\left(x, t, T_{n}\left(u_{n}\right)\right) \longrightarrow a(x, t, u) \text { a.e. in } \mathrm{Q} .
$$

Therefore, (3.10) and the Egorov theoren imply that for the above $\delta>0$, there exists a measurable subset $Q_{\delta} \subset Q$ such that $\left|Q-Q_{\delta}\right| \leq \delta$ and

$$
a\left(x, t, T_{n}\left(u_{n}\right)\right) \longrightarrow a(x, t, u) \text { uniformly on } Q_{\delta} .
$$


Namely, for the above $\varepsilon>0$, there exists a large nature number $N_{0}$ such that for every $n>N_{0}$,

$$
\left|a\left(x, t, T_{n}\left(u_{n}\right)\right)-a(x, t, u)\right|<\frac{\varepsilon}{3 C_{13}\|D \psi\|_{L^{q^{\prime}}(Q)}}, \quad \forall x \in Q_{\delta} .
$$

By (3.1), there is a large nature number $N_{1} \geq N_{0}$ such that for every $n>N_{1}$,

$$
\left|\int_{Q} a(x, t, u)\left(D u_{n}-D u\right) D \psi d x d t\right|<\frac{\varepsilon}{3} \text {. }
$$

On one hand, we have

$$
\begin{aligned}
& \int_{Q}\left[a\left(x, t, T_{n}\left(u_{n}\right)\right) D u_{n}-a(x, t, u) D u\right] D \psi d x d t \\
& =\int_{Q_{\delta}}\left[a\left(x, t, T_{n}\left(u_{n}\right)\right)-a(x, t, u)\right] D u_{n} D \psi d x d t \\
& \quad+\int_{Q-Q_{\delta}}\left[a\left(x, t, T_{n}\left(u_{n}\right)\right)-a(x, t, u)\right] D u_{n} D \psi d x d t \\
& \quad+\int_{Q} a(x, t, u)\left(D u_{n}-D u\right) D \psi d x d t .
\end{aligned}
$$

On the other hand, (3.12), Hölder's inequality and (2.45) imply that for every $n>N_{0}$,

$$
\begin{aligned}
& \left|\int_{Q_{\delta}}\left[a\left(x, t, T_{n}\left(u_{n}\right)\right)-a(x, t, u)\right] D u_{n} D \psi d x d t\right| \\
& <\frac{\varepsilon}{3 C_{13}\|D \psi\|_{L^{q^{\prime}}(Q)}}\left\|D u_{n}\right\|_{L^{q}(Q)}\|D \psi\|_{L^{q^{\prime}}(Q)} \leq \frac{\varepsilon}{3} .
\end{aligned}
$$

Using Hölder's inequality, (2.45), (3.6) and (1.1), let $E=Q-Q_{\delta}$ in (3.9), we obtain

$$
\begin{aligned}
& \left|\int_{Q-Q_{\delta}}\left[a\left(x, t, T_{n}\left(u_{n}\right)\right)-a(x, t, u)\right] D u_{n} D \psi d x d t\right| \\
& \leq 2 \beta\left\|D u_{n}\right\|_{L^{q}(Q)}\|D \psi\|_{L^{q^{\prime}}\left(Q-Q_{\delta}\right)}<2 \beta C_{13} \frac{\varepsilon}{6 \beta C_{13}}=\frac{\varepsilon}{3}, \quad \forall n .
\end{aligned}
$$

Thus (3.13)-(3.16) imply that for all $\varepsilon>0$, there exists a large natural number $N_{1}$ such that for every $n>N_{1}$,

$$
\left|\int_{Q}\left[a\left(x, t, T_{n}\left(u_{n}\right)\right) D u_{n}-a(x, t, u) D u\right] D \psi d x d t\right|<\frac{\varepsilon}{3}+\frac{\varepsilon}{3}+\frac{\varepsilon}{3}=\varepsilon .
$$

Hence

$$
\left.\int_{Q} a\left(x, t, T_{n}\left(u_{n}\right)\right) D u_{n} D \psi d x d t \longrightarrow \int_{Q} a(x, t, u)\right) D u D \psi d x d t .
$$

Thus we obtain $u$ is a solution to problem $(P)$ in the sense of distributions. The proof of Theorem 1.4 is finished.

Proof of Theorem 1.5. Let

$$
h_{k}(s)=1-\left|T_{1}\left(s-T_{k}(s)\right)\right|, \quad H_{k}(s)=\int_{0}^{s} h_{k}(\tau) d \tau, \quad \forall s \in R, \quad \forall k>0 .
$$


If we multiply the approximate equation of problem $\left(P_{n}\right)$ by $h_{k}\left(u_{n}\right)$, we get in the sense of distributions

$$
\begin{aligned}
\left(H_{k}\left(u_{n}\right)\right)_{t}= & \operatorname{div}\left(h_{k}\left(u_{n}\right) a\left(x, t, T_{n}\left(u_{n}\right)\right) D u_{n}\right) \\
& -a\left(x, t, T_{n}\left(u_{n}\right)\right) D u_{n} D u_{n} h_{k}^{\prime}\left(u_{n}\right)+f_{n} h_{k}\left(u_{n}\right) .
\end{aligned}
$$

Note that $\operatorname{supp}\left(h_{k}\right) \subseteq[-k-1, k+1], 0 \leq h_{k} \leq 1,\left|h_{k}^{\prime}\right| \leq 1$, if $n>k+1$,

$$
\begin{aligned}
h_{k}\left(u_{n}\right) a\left(x, t, T_{n}\left(u_{n}\right)\right) D u_{n} & =h_{k}\left(u_{n}\right) a\left(x, t, T_{k+1}\left(u_{n}\right)\right) D T_{k+1}\left(u_{n}\right), \\
a\left(x, t, T_{n}\left(u_{n}\right)\right) D u_{n} D u_{n} h_{k}^{\prime}\left(u_{n}\right) & =a\left(x, t, T_{k+1}\left(u_{n}\right)\right) D T_{k+1}\left(u_{n}\right) D T_{k+1}\left(u_{n}\right) h_{k}^{\prime}\left(u_{n}\right) .
\end{aligned}
$$

By Lemma 2.8, (2.1) and the above equalities, for fixed $k>0$, we can deduce that $h_{k}\left(u_{n}\right) a\left(x, t, T_{n}\left(u_{n}\right)\right) D u_{n}$ is bounded in $L^{2}(Q)$, and $a\left(x, t, T_{n}\left(u_{n}\right)\right) D u_{n} D u_{n} h_{k}^{\prime}\left(u_{n}\right)$ is bounded in $L^{1}(Q)$. Hence $\left(H_{k}\left(u_{n}\right)\right)_{t}$ is bounded in $L^{2}\left(0, T ; H^{-1}(\Omega)\right)+L^{1}(Q)$, thus there exists some $s>1$ such that $\left(H_{k}\left(u_{n}\right)\right)_{t}$ is bounded in $L^{1}\left(0, T ; H^{-s}(\Omega)\right)$. By virtue of $D H_{k}\left(u_{n}\right)=h_{k}\left(u_{n}\right) D u_{n}=h_{k}\left(u_{n}\right) D T_{k+1}\left(u_{n}\right)$, (2.60) implies that $H_{k}\left(u_{n}\right)$ is bounded in $L^{2}\left(0, T ; H_{0}^{1}(\Omega)\right)$. Hence a compactness result (see [27]) allows to conclude that $H_{k}\left(u_{n}\right)$ is compact in $L^{1}(Q)$. By Theorem 1.1 in [23], we have $H_{k}\left(u_{n}\right) \in$ $C\left([0, T] ; L^{1}(\Omega)\right)$. Thus there exists a subsequence of $\left\{H_{k}\left(u_{n}\right)\right\}$ (still be denoted by $\left.\left\{H_{k}\left(u_{n}\right)\right\}\right)$ such that it also converges in measure and almost everywhere in $Q$.

For all $\sigma>0$ and $\varepsilon>0$, we have

$$
\begin{aligned}
\operatorname{meas}\left\{\left|u_{n}-u_{m}\right|>\sigma\right\} \leq & \operatorname{meas}\left\{\left|u_{n}\right|>k\right\}+\operatorname{meas}\left\{\left|u_{m}\right|>k\right\} \\
& +\operatorname{meas}\left\{\left|H_{k}\left(u_{n}\right)-H_{k}\left(u_{m}\right)\right|>\sigma\right\} .
\end{aligned}
$$

By (2.61) in Lemma 2.8, we can choose $k$ large enough to have

$$
\operatorname{meas}\left\{\left|u_{n}\right|>k\right\}+\operatorname{meas}\left\{\left|u_{m}\right|>k\right\}<\frac{\varepsilon}{2}, \quad \forall n, m .
$$

Furthermore, for the above fixed $k$, we can choose a large $\bar{N}$ such that

$$
\operatorname{meas}\left\{\left|H_{k}\left(u_{n}\right)-H_{k}\left(u_{m}\right)\right|>\sigma\right\}<\frac{\varepsilon}{2}, \quad \forall n, m>\bar{N} .
$$

(3.22) and (3.23) yield

$$
\operatorname{meas}\left\{\left|u_{n}-u_{m}\right|>\sigma\right\}<\varepsilon, \quad \forall n, m>\bar{N} .
$$

Now (3.24) implies that $\left\{u_{n}\right\}$ is a Cauchy sequence in measure in $Q$. Hence there exists a measurable function $u$ such that

$$
u_{n} \longrightarrow u \text { a.e. in } \mathrm{Q} \text {. }
$$

Thus we get

$$
H_{k}\left(u_{n}\right) \longrightarrow H_{k}(u) \text { a.e. in } Q .
$$

Since $\left|H_{k}\right| \leq k+1$, (3.26) and Lebesgue's dominated convergence theorem yield

$$
H_{k}\left(u_{n}\right) \longrightarrow H_{k}(u) \text { strongly in } L^{2}(Q) \text {. }
$$

Since $H_{k}\left(u_{n}\right)$ is bounded in $L^{2}\left(0, T ; H_{0}^{1}(\Omega)\right)$ and noting that (3.27) holds, we have

$$
H_{k}\left(u_{n}\right) \longrightarrow H_{k}(u) \text { weakly in } L^{2}\left(0, T ; H_{0}^{1}(\Omega)\right) \text {. }
$$

Now (3.25) yields

$$
T_{k}\left(u_{n}\right) \longrightarrow T_{k}(u) \text { a.e. in } Q \text {. }
$$

Using Lebesgue's dominated convergence theorem once again, we get

$$
T_{k}\left(u_{n}\right) \longrightarrow T_{k}(u) \text { strongly in } L^{2}(Q) \text {. }
$$


From (2.60) and (3.30), it follows that

$$
T_{k}\left(u_{n}\right) \longrightarrow T_{k}(u) \text { weakly in } L^{2}\left(0, T ; H_{0}^{1}(\Omega)\right) .
$$

Then (2.59), (3.25) and Fatou's lemma yield $u \in L^{\infty}\left(0, T ; L^{1}(\Omega)\right)$.

Similarly to Theorem 2.1 in [23], we can prove

$$
T_{k}\left(u_{n}\right) \longrightarrow T_{k}(u) \text { strongly in } L^{2}\left(0, T ; H_{0}^{1}(\Omega)\right) .
$$

Hence

$$
D T_{k}\left(u_{n}\right) \longrightarrow D T_{k}(u) \text { a.e. in } Q \text {. }
$$

Choosing $T_{1}\left(u_{n}-T_{k}\left(u_{n}\right)\right)$ as a test function for problem $\left(P_{n}\right)$, we can obtain

$$
\int_{\Omega} \tilde{T}\left(u_{n}(T)\right) d x+\int_{\left\{k<\left|u_{n}\right| \leq k+1\right\}} a\left(x, t, T_{n}\left(u_{n}\right)\right)\left|D u_{n}\right|^{2} d x d t \leq \int_{\left\{\left|u_{n}\right| \geq k\right\}}\left|f_{n}\right| d x d t
$$

where $\tilde{T}\left(u_{n}(T)\right)=\int_{0}^{u_{n}(T)} T_{1}\left(s-T_{k}(s)\right) d s$.

It is easy to see that $\tilde{T}\left(u_{n}(T)\right) \geq 0$ a.e. in $\Omega$. Hence we have

$$
\int_{\left\{k<\left|u_{n}\right| \leq k+1\right\}} a\left(x, t, T_{n}\left(u_{n}\right)\right)\left|D u_{n}\right|^{2} d x d t \leq \int_{\left\{\left|u_{n}\right| \geq k\right\}}\left|f_{n}\right| d x d t .
$$

Letting $n \rightarrow \infty$ in (3.35) and using Fatou's lemma and Vitali's theorem on the left side and right side of (3.35) respectively, we get

$$
\left.\int_{\{k<|u| \leq k+1\}} a(x, t, u)\right)|D u|^{2} d x d t \leq \int_{\{|u| \geq k\}}|f| d x d t .
$$

Thus from (3.36) we can deduce that

$$
\left.\lim _{k \rightarrow \infty} \int_{\{k<|u| \leq k+1\}} a(x, t, u)\right)|D u|^{2} d x d t=0 .
$$

Then (3.25), (3.29), (3.32) and Vitali's theorem imply that

$$
h_{k}\left(u_{n}\right) a\left(x, t, T_{n}\left(u_{n}\right)\right) D u_{n} \longrightarrow h_{k}(u) a\left(x, t, T_{k+1}(u)\right) D T_{k+1}(u)
$$

strongly in $L^{2}(Q)$ and

$$
a\left(x, t, T_{n}\left(u_{n}\right)\right) D u_{n} D u_{n} h_{k}^{\prime}\left(u_{n}\right) \longrightarrow a\left(x, t, T_{k+1}(u)\right) D T_{k+1}(u) D T_{k+1}(u) h_{k}^{\prime}(u)
$$

strongly in $L^{1}(Q)$. Let $n \rightarrow \infty$ in (3.20). We obtain in the sense of distributions that

$$
\begin{aligned}
\left(H_{k}(u)\right)_{t}= & \operatorname{div}\left(h_{k}(u) a\left(x, t, T_{k+1}(u)\right) D T_{k+1}(u)\right) \\
& -a\left(x, t, T_{k+1}(u)\right) D T_{k+1}(u) D T_{k+1}(u) h_{k}^{\prime}(u)+f h_{k}(u) .
\end{aligned}
$$

Hence $\left(H_{k}(u)\right)_{t} \in L^{2}\left(0, T ; H^{-1}(\Omega)\right)+L^{1}(Q)$. By Theorem 1.1 in [23], we also have $H_{k}(u) \in C\left([0, T] ; L^{1}(\Omega)\right)$. Since $H_{k}\left(u_{n}(0)\right)=0$, thus we get $H_{k}(u(0))=0$. For every $\phi \in L^{2}\left(0, T ; H_{0}^{1}(\Omega)\right) \cap L^{\infty}(Q)$ such that $\phi_{t} \in L^{2}\left(0, T ; H^{-1}(\Omega)\right)+L^{1}(Q)$ and for all $\tau \in(0, T]$, using $T_{l}\left(H_{k}(u)-\phi\right) \chi_{(0, \tau)}(t)$ as a test function in (3.40), and integrating 
by parts we obtain

$$
\begin{aligned}
& \int_{\Omega} S_{l}\left(H_{k}(u)-\phi\right)(\tau) d x-\int_{\Omega} S_{l}(-\phi(0)) d x+\int_{0}^{\tau}\left\langle\phi_{t}, T_{l}\left(H_{k}(u)-\phi\right)\right\rangle d t \\
& \quad+\int_{0}^{\tau} \int_{\Omega} h_{k}(u) a\left(x, t, T_{k+1}(u)\right) D T_{k+1}(u) D T_{l}\left(H_{k}(u)-\phi\right) d x d t \\
& \quad+\int_{0}^{\tau} \int_{\Omega} a\left(x, t, T_{k+1}(u)\right) D T_{k+1}(u) D T_{k+1}(u) h_{k}^{\prime}(u) T_{l}\left(H_{k}(u)-\phi\right) d x d t \\
& =\int_{0}^{\tau} \int_{\Omega} f h_{k}(u) T_{l}\left(H_{k}(u)-\phi\right) d x d t .
\end{aligned}
$$

Noting that if $k \rightarrow \infty$, we have

$$
h_{k}(u) \longrightarrow 1 \text { a.e. in } Q
$$

and

$$
H_{k}(u) \longrightarrow u \text { a.e. in } Q \text {. }
$$

Since $h_{k}^{\prime}(u)=-\operatorname{sign}(u) \chi_{\{k \leq|u| \leq k+1\}}, \operatorname{sign}\left(H_{k}(u)\right)=\operatorname{sign}(u)$, and $\left|H_{k}(u)\right|>k$ if $|u|>$ $k$; and $H_{k}(u)=u$ if $|u| \leq k$. Moreover, if $\left|H_{k}(u)\right|>l+\|\phi\|_{L^{\infty}(Q)}=L$, we have $D T_{l}\left(H_{k}(u)-\phi\right)=0$. Hence if $k>l+\|\phi\|_{L^{\infty}(Q)}$, Thus we have

$$
\begin{aligned}
& \int_{0}^{\tau} \int_{\Omega} h_{k}(u) a\left(x, t, T_{k+1}(u)\right) D T_{k+1}(u) D T_{l}\left(H_{k}(u)-\phi\right) d x d t \\
& =\int_{0}^{\tau} \int_{\Omega} a\left(x, t, T_{L}(u)\right) D T_{L}(u) D T_{l}\left(T_{L}(u)-\phi\right) d x d t .
\end{aligned}
$$

It follows from (3.37) that

$$
\lim _{k \rightarrow \infty} \int_{0}^{\tau} \int_{\Omega} a\left(x, t, T_{k+1}(u)\right) D T_{k+1}(u) D T_{k+1}(u) h_{k}^{\prime}(u) T_{l}\left(H_{k}(u)-\phi\right) d x d t=0 .
$$

Lebesgue's dominated convergence theorem and (3.42)-(3.43) imply that

$$
\lim _{k \rightarrow \infty} \int_{0}^{\tau} \int_{\Omega} f h_{k}(u) T_{l}\left(H_{k}(u)-\phi\right) d x d t=\int_{0}^{\tau} \int_{\Omega} f T_{l}(u-\phi) d x d t .
$$

We can also prove if $k \rightarrow \infty$,

$$
T_{l}\left(H_{k}(u)-\phi\right) \longrightarrow T_{l}(u-\phi) \quad \text { strongly in } L^{2}\left(0, T ; H_{0}^{1}(\Omega)\right)
$$

and

$$
T_{l}\left(H_{k}(u)-\phi\right) \longrightarrow T_{l}(u-\phi) \quad \text { weak }^{*} \text { in } L^{\infty}(\Omega) .
$$

From (3.47) and (3.48) we get

$$
\lim _{k \rightarrow \infty} \int_{0}^{\tau}\left\langle\phi_{t}, T_{l}\left(H_{k}(u)-\phi\right)\right\rangle d t=\int_{0}^{\tau}\left\langle\phi_{t}, T_{l}(u-\phi)\right\rangle d t
$$

Since for a.e. $\tau \in[0, T]$, a.e. $x \in \Omega$,

$$
\left|H_{k}(u)\right| \leq|u|, \quad 0 \leq S_{l}\left(H_{k}(u)-\phi\right)(\tau) \leq l[|u(\tau)|+|\phi(\tau)|]
$$

combining with $u \in L^{\infty}\left(0, T ; L^{1}(\Omega)\right)$ and $\phi \in C\left([0, T] ; L^{1}(\Omega)\right)$, by Lebesgue's dominated convergence theorem and (3.43), we get

$$
\lim _{k \rightarrow \infty} \int_{\Omega} S_{l}\left(H_{k}(u)-\phi\right)(\tau) d x=\int_{\Omega} S_{l}(u-\phi)(\tau) d x
$$


Now (3.44)-(3.46), (3.49)-(3.50) and (3.41) yield for a.e. $\tau \in[0, T]$,

$$
\begin{aligned}
& \int_{\Omega} S_{l}(u-\phi)(\tau) d x-\int_{\Omega} S_{l}(-\phi(0)) d x+\int_{0}^{\tau}\left\langle\phi_{t}, T_{l}(u-\phi)\right\rangle d t \\
& +\int_{0}^{\tau} \int_{\Omega} a(x, t, u) D u D T_{l}(u-\phi) d x d t=\int_{0}^{\tau} \int_{\Omega} f T_{l}(u-\phi) d x d t .
\end{aligned}
$$

This shows that the first term on the left side of the above equality is almost everywhere equal to a continuous function on $[0, T]$. Replacing $l$ with $k$ in (3.51), we obtain (2.1)-(2.2) and $u$ is an entropy solution to problem $(P)$. By (2.61), we have

$$
\int_{Q} \chi_{\left\{\left|u_{n}\right|>k\right\}} d x d t=\operatorname{meas}\left\{\left|u_{n}\right|>k\right\} \leq \frac{C_{19}}{k^{r}} .
$$

Thus (3.25), (3.52) and Fatou's lemma yield

$$
\operatorname{meas}\{|u|>k\}=\int_{Q} \chi_{\{|u|>k\}} d x d t \leq \frac{C_{19}}{k^{r}} .
$$

Rewriting (3.43) as follows

$$
k \text { meas }\{|u|>k\}^{\frac{1}{r}} \leq C_{19}^{\frac{1}{r}} .
$$

Thus by Definition 2.2, we obtain $u \in \mathcal{M}^{r}(Q)$.

To complete the proof of (1.11), we need to prove

$$
D u_{n} \longrightarrow D u \quad \text { a.e. in } Q \text {. }
$$

In fact, for all $\sigma>0$ and $\varepsilon>0$, we have

$$
\begin{aligned}
\operatorname{meas}\left\{\left|D u_{n}-D u\right|>\sigma\right\} \leq & \operatorname{meas}\left\{\left|u_{n}\right|>k\right\}+\operatorname{meas}\{|u|>k\} \\
& +\operatorname{meas}\left\{\left|D T_{k}\left(u_{n}\right)-D T_{k}(u)\right|>\sigma\right\} .
\end{aligned}
$$

By (2.61) in Lemma 2.8 and (1.10), we can choose $k$ large enough to have

$$
\operatorname{meas}\left\{\left|u_{n}\right|>k\right\}+\operatorname{meas}\{|u|>k\}<\frac{\varepsilon}{2}, \quad \forall n .
$$

For the above $k,(3.33)$ implies that there exists a large $\bar{N}$ such that

$$
\operatorname{meas}\left\{\left|D T_{k}\left(u_{n}\right)-D T_{k}(u)\right|>\sigma\right\}<\frac{\varepsilon}{2}, \quad \forall n>\bar{N} .
$$

Now (3.57) and (3.58) yield

$$
\text { meas }\left\{\left|D u_{n}-D u\right|>\sigma\right\}<\varepsilon, \quad \forall n>\bar{N} .
$$

Hence from (3.54), we can deduce that (3.55) (up to a subsequence) holds. Similarly to (3.52)-(3.54), by (2.62) and (3.55), we obtain $|D u| \in \mathcal{M}^{q}(Q)$. Thus the proof of Theorem 1.5 is completed.

\section{References}

[1] Alvino, A., L. Boccardo, V. Ferone, L. Orsina, and G. Trombetti: Existence results for nonlinear elliptic equations with degenerate coercivity. - Ann. Mat. Pura Appl. (4) 182:1, 2003, 53-79.

[2] Alvino, A., V. Ferone, and G. Trombetti: A priori estimates for a class of non-uniformly elliptic equations. - Atti Sem. Mat. Fis. Univ. Modena Reggio Emilia 46, 1998, 381-391. 
[3] Andreu, F., J. M. Mazon, S. Segura de Leon, and J. Toledo: Existence and uniquence for a degenerate parabolic equation with $L^{1}$ data. - Trans. Amer. Math. Soc. 351:1, 1999, 285-306.

[4] Aronson, D. G., and J. SERrin: Local behavior of solutions of quasilinear parabolic equations. - Arch. Ration. Mech. Anal. 25, 1967, 81-122.

[5] Baras, P., and M. Pierre: Problèmes paraboliques semi-linéaires avec données mesures. Appl. Anal. 18:1-2, 1984, 111-149.

[6] Bénilan, Ph., L. Boccardo, T. Gallouët, R. Gariepy, M. Pierre, and J. L. Vazquez: An $L^{1}$-theory of existence and uniqueness of solutions of non-linear elliptic equations. - Ann. Sc. Norm. Super. Pisa Cl. Sci. (4) 22:2, 1995, 240-273.

[7] Bénilan, Ph., H. Brezis, and M. G. Crandall: A semilinear equation in $L^{1}$. - Ann. Sc. Norm. Super. Pisa Cl. Sci. (4) 2, 1975, 523-555.

[8] Bocchrdo, L.: Existence and regularity results for quasilinear parabolic equations. - In: Reaction diffusion systems (Trieste, 1995), Lect. Notes Pure Appl. Math. 194, Dekker, New York, 1998, 51-58

[9] Boccardo, L.: Some elliptic problems with degenerate coercivity. - Adv. Nonlinear Stud. 6:1, 2006, 1-12.

[10] Boccardo, L., and H. Brezis: Some remarks on a class of elliptic equations with degenerate coercivity. - Boll. Unione Mat. Ital. Sez. B Artic. Ric. Mat. (8) 6:3, 2003, 521-530.

[11] Boccardo, L., A. Dall'Aglio, T. Gallouët, and L. Orsina: Nonlinear parabolic equations with measure data. - J. Funct. Anal. 147:1, 1997, 237-258.

[12] Bocchrdo, L., A. Dall'Aglio, T. Gallouët, and L. Orsina: Existence and regularity results for some nonlinear parabolic equations. - Adv. Math. Sci. Appl. 9:2, 1999, 1017-1031.

[13] Bocchrdo, L., A. Dall'Aglio, and L. Orsina: Existence and regularity results for some elliptic equations with degenerate coercivity. - Atti Semin. Mat. Fis. Univ. Modena Reggio Emilia 46, 1998, 51-81.

[14] Boccardo, L., T. GallouËT: Nonlinear elliptic and parabolic equations involving measure data. - J. Funct. Anal. 87:1, 1989, 149-169.

[15] Dall'Aglio, A., and L. Orsina: Existence results for some nonlinear parabolic equations with nonregular data. - Differential Integral Equations 5:6, 1992, 1335-1354.

[16] Di Benedetto, E.: Degenerate parabolic equations. - Springer-Verlag, New York, 1993.

[17] Droniou, J., and A. Prignet: Equivalence between entropy and renormalized solutions for parabolic equations with smooth measure data. - NoDEA Nonlinear Differential Equations Appl. 14:1-2, 2007, 181-205.

[18] Giachetti, D., and M. M. Porzio: Existence results for some nonuniformly elliptic equations with irregular data. - J. Math. Anal. Appl. 257:1, 2001, 100-130.

[19] Giachetti, D., and M. M. Porzio: Elliptic equations with degenerate coercivity: gradient regularity. - Acta Math. Sin. (Engl. Ser.) 19:2, 2003, 349-370.

[20] Ladyženskaja, O. A., V. A. Solonnikov, and N. N. Ural'Ceva: Linear and quasilinear equations of parabolic type. - Translations of Amer. Math. Soc., Amer. Math. Soc. Providence, 1968.

[21] Li, F.: Existence of entropy solutions to some parabolic problems with $L^{1}$ data. - Acta. Math. Sinica (Chin. Ser.) 18:1, 2002, 119-128.

[22] Lions, J. L.: Quelques méthodes de résolution des problémes aux limites nonlinéaires. - Dunod, Paris, 1969.

[23] Porretta, A.: Existence results for nonlinear parabolic equations via strong convergence of truncations. - Ann. Mat. Pura. Appl. 177, 1999, 143-172. 
[24] Porzio, M. M., and M. A. Pozio: Parabolic equations with non-linear, degenerate and spacetime dependent operators. - J. Evol. Equ. 8, 2008, 31-70.

[25] Prignet, A.: Existence and uniqueness of "entropic" solutions of parabolic problems with $L^{1}$ data. - Nonlinear Anal. 28:12, 1997, 1943-1954.

[26] Segura de León, S., and J. Toledo Regularity for entropy solutions of parabolic PLaplacian equations. - Publ. Mat. 43, 1999, 665-683.

[27] Simon, J.: Compact sets in the space $L^{p}(0, T ; B)$. - Ann. Mat. Pura. Appl. 146, 1987, 65-96.

[28] Ziemer, W. P.: Weakly differentiable functions. - Springer-Verlag, New York, 1989.

Received 18 April 2012 